\title{
Advanced Small Modular Reactor (SMR) Probabilistic Risk Assessment (PRA) Demonstration
}

Curtis Smith Steven Prescott Tony Koonce

April 2014

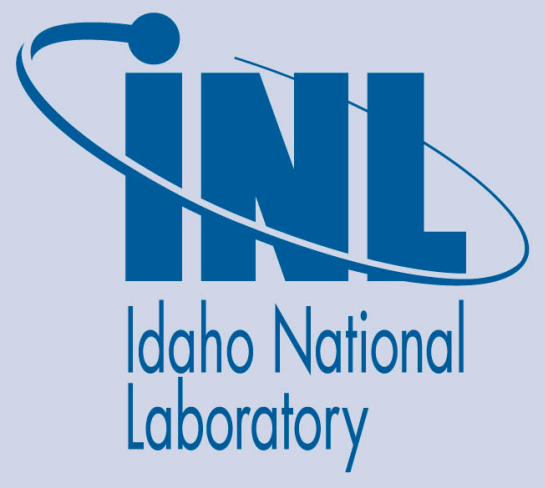

The INL is a U.S. Department of Energy National Laboratory operated by Battelle Energy Alliance 
INL/EXT-14-31876

\title{
Advanced Small Modular Reactor (SMR) Probabilistic Risk Assessment (PRA) Demonstration
}

\author{
Curtis Smith \\ Steven Prescott \\ Tony Koonce
}

April 2014

Idaho National Laboratory
Idaho Falls, Idaho 83415

http://www.inl.gov

Prepared for the

U.S. Department of Energy

Office of Nuclear Energy

Under DOE Idaho Operations Office

Contract DE-AC07-05ID14517 


\section{Advanced Small Modular Reactor (SMR) Probabilistic Risk Assessment (PRA) Demonstration}

Describing the framework to support the implementation of a state-of-the-art PRA to represent advanced flooding simulation capabilities for SMRs

Curtis Smith

Steven Prescott

Tony Koonce 


\section{Table of Contents}

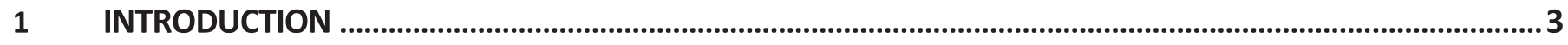

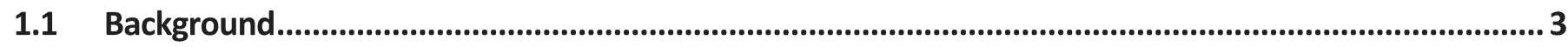

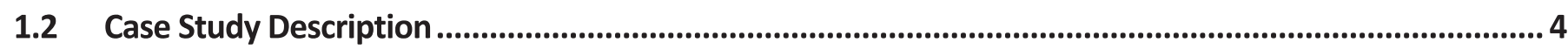

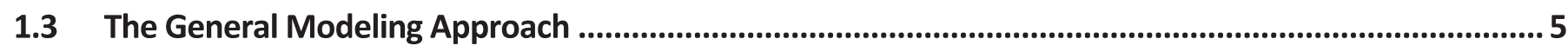

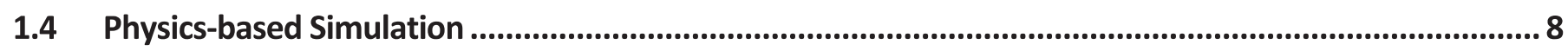

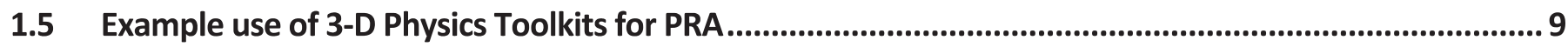

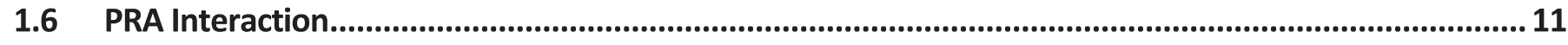

2.1 Bayesian Modeling for Extreme Event Initiators ................................................................................... 12

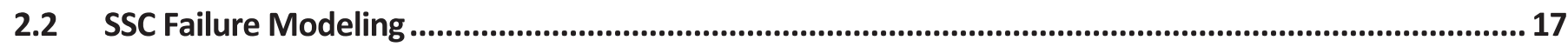

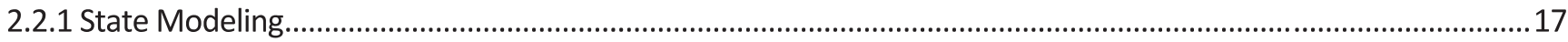

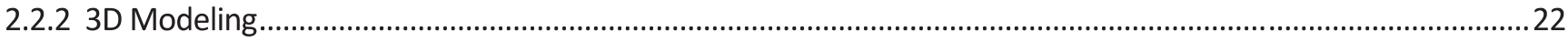

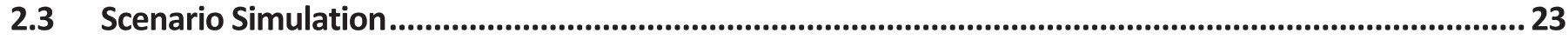

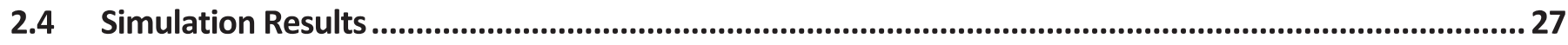

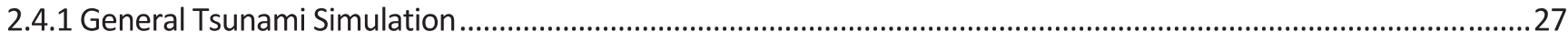

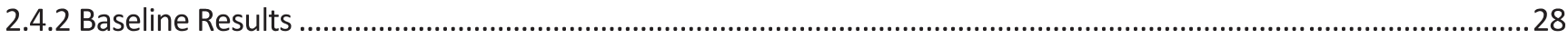

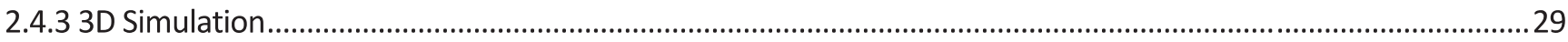

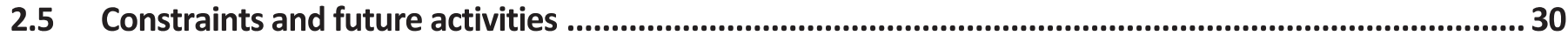

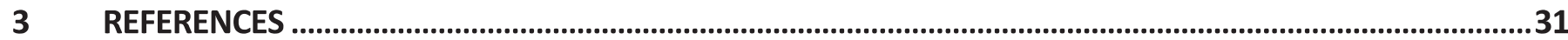




\section{Chapter}

\section{Introduction}

\subsection{Background}

A key area of the Advanced Small Modular Reactor (SMR) Probabilistic Risk Assessment (PRA) strategy is the development of methodologies and tools that will be used to predict the safety, security, safeguards, performance, and deployment viability of SMRs. The goal of the SMR PRA activity will be to develop quantitative methods and tools and the associated analysis framework for assessing a variety of risks.

Development and implementation of SMR-focused safety assessment methods may require new analytic methods or adaptation of traditional methods to the advanced design and operational features of SMRs. We will need to move beyond the current limitations such as static, logic-based models in order to provide more integrated, time-based, and scenariobased models where predictive modeling are tied to causal factors. The development of SMR-specific models for safety margin determination will provide a safety case that describes potential accidents, design options (including postulated controls), possible vulnerabilities to be addressed, and supports licensing activities by providing a technical basis for the safety envelope.

For the next generation of nuclear power plants (NPPs), it is imperative that safety analysis technologies evolve into an accepted, encompassing, validated, and integral part of the plant in order to reduce costs and to demonstrate safe operation. Currently, a variety of stated (and unstated) and substantiated (and unsubstantiated) assumptions have been made for SMR plant designs, including:

- Greater safety margins

- Smaller exclusions zone (the traditional zone is too large)

- Simpler emergency planning

- Reliance on passive safety systems

- Smaller and delayed source term

- Accident doses will be lower

These and other statements may be accurate, but they will need to be substantiated as part of a risk-informed approach. During FY2013, Idaho National Laboratory (INL) proposed an approach to expand and advance the state-of-the-practice in PRA [1]:

Develop a framework for applying modern computational tools to create advanced risk-based methods for identifying design vulnerabilities in SMRs. This framework will require the fusion of state-of-the-art PRA methods, advanced $3 D$ visualization methods, and high-performance optimization within a flexible open source framework. An initial effort will be to define the conceptual framework and a draft implementation plan. 
This report documents the progress that was made to implement the PRA framework, specifically by way of demonstration of an advanced 3D approach to representing, quantifying and understanding flooding risks to a nuclear power plant.

\subsection{Case Study Description}

The task that is described in this report is for the deliverable:

"Complete a prototype demonstration of an external hazard (such as flooding) impacting a hypothetical plant facility"

This deliverable supports the "Framework Development for Phenomena Representation" as part of the INL PRA Methodology for SMRs project.

The case study described in this report, looking at a flooding hazard, focuses on a riskinformed approach to represent meaningful (i.e., representative facility hazards) scenarios and consequences by using an advanced 3D SMR representation. This approach (as we will describe) does:

- Identify, model and analyze the appropriate physics (i.e., the physics that needs to be included to determine plant vulnerabilities) in a scenario-based fashion.

- Manage the communication and interactions between different physics modeling and analysis technologies.

- $\quad$ Determine what facets of the problem are important for measuring and managing risk using this new application.

- $\quad$ Provide an analysis platform that can be used to "virtual stress-test" different riskinformed strategies.

- Manage the computational infrastructure related to facility representation, scenario depiction, and physics prediction.

- $\quad$ Identify what behaviors the physics-models should simulate.

A notional depiction of these attributes of the proposed approach is shown in Figure 1. The approach has several defining attributes focused within three general areas:

1. Models - A single 3D representation of all key systems, structures, and components (SSCs) to be defined for a particular facility. We will be able to simulate with these models - by understanding how each SSC interacts with other parts of the facility (e.g., failure dependencies) - the hazard-induced susceptibilities of each SSC (e.g., energy from a seismic event may fail a component, flooding may fail a component), and how to "dial up" model fidelity/resolution when needed (e.g., if flooding occurs in a room, the behavior of components related to water egress would become more important or enabled).

2. Phenomena - An approach to effectively representing hazards and their effect on physical behavior at a facility will need to be determined. In many cases, multiple models of a specific phenomenon may be available, but this ensemble of models will need to be intelligently managed. For example, how spatial effects may drive a 
scenario in an undesired fashion (e.g., a pipe break caused by a seismic event may flood a pump room) could be determined using several different methods consequently we will need to be able to internally resolve and weight the variety of possible internal-analysis results.

3. Integration - Any advanced risk-informed decision support approach will rely on a variety of probabilistic and mechanistic information. The safety, security, and economic drivers will need to be integrated in order to determine the effectiveness of proposed mitigation strategies (e.g., should we install a sea-wall?). We will need to be able to manage all (important) hazards for all (important) scenarios all of the time the facility is in operation.

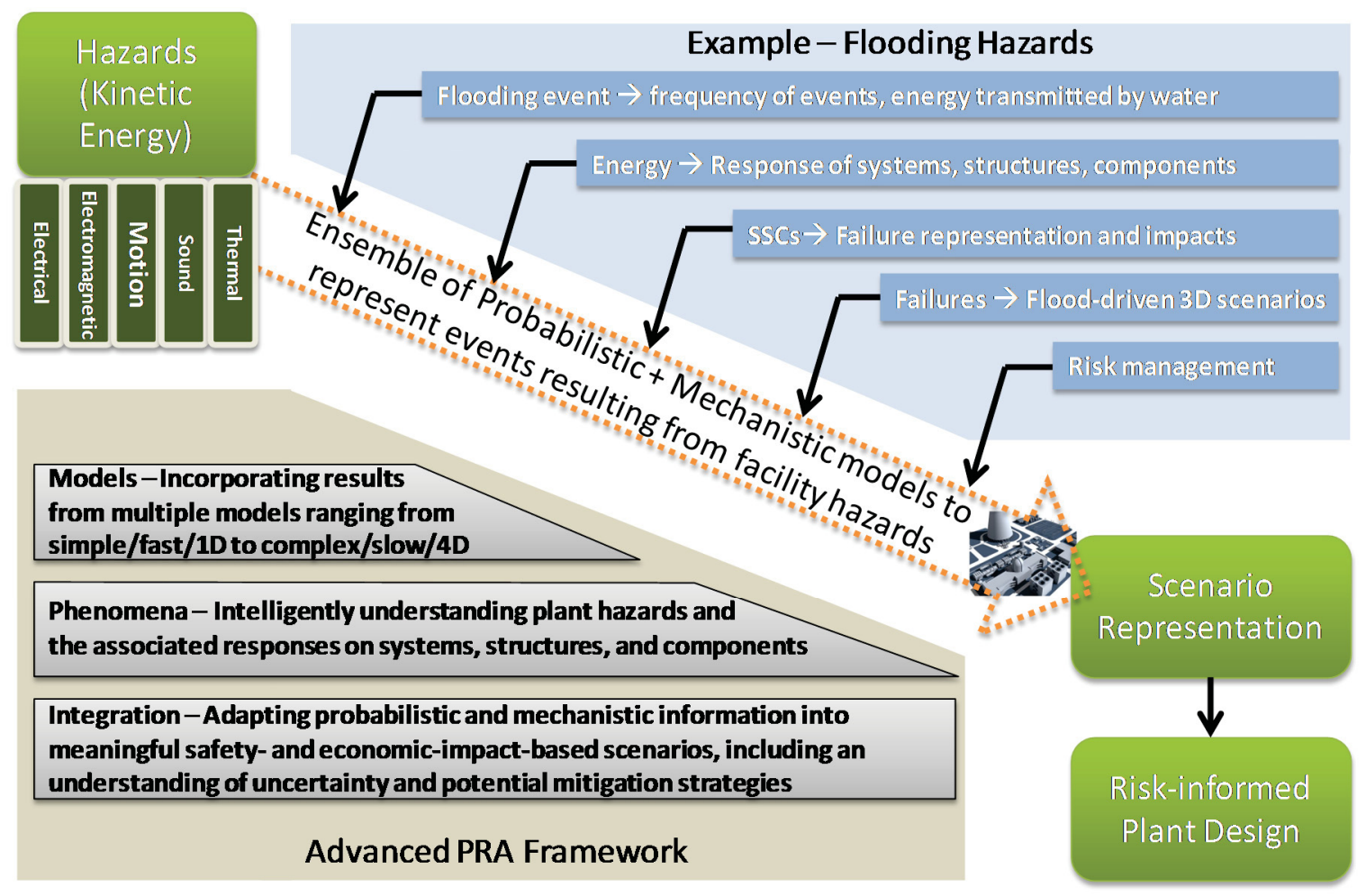

Figure 1. High-level features of the proposed advanced SMR PRA approach.

Chapter 1 of this report provides background information on the general approach that is used for the advanced PRA. Chapter 2 provides details on the flooding case study.

\subsection{The General Modeling Approach}

In order to enable probabilistic aspects of nuclear-based systems, we will be developing a variety of infrastructure methods/models based upon simulation. The INL has extensive capability in classical PRA, but for future applications, the proposed development is superior to the static logic-based approaches used in existing accident risk analysis, in which 
simplifications and numerical approximations are necessary. Successfully linking probabilistic system simulation to system physics is a facet of advanced SMR PRA methods and will directly address problems such as highly time-dependent scenarios in SMR risk analysis, where probability is a key aspect of the scenario.

Note that the science and engineering communities are increasingly moving to more sophisticated mechanistic models in order to better represent the complexities of "the real world." As part of this movement, we find an increasing reliance on or the need for probabilistic approaches, including the elicitation of information. The application of probability concepts is needed to support the use of mechanistic models in a probabilistic world.

Capturing what, why, and how one knows something related to science and engineering is important to realizing the potential of complex nuclear systems.

A high-level depiction of the major modules that would be required in the cloud-based PRA approach is shown in Figure 2. As shown in the figure, analysts and reviewers would access the tools and PRA models by using an Internet browser. The other modules provide the modeling and analysis capabilities for the user.

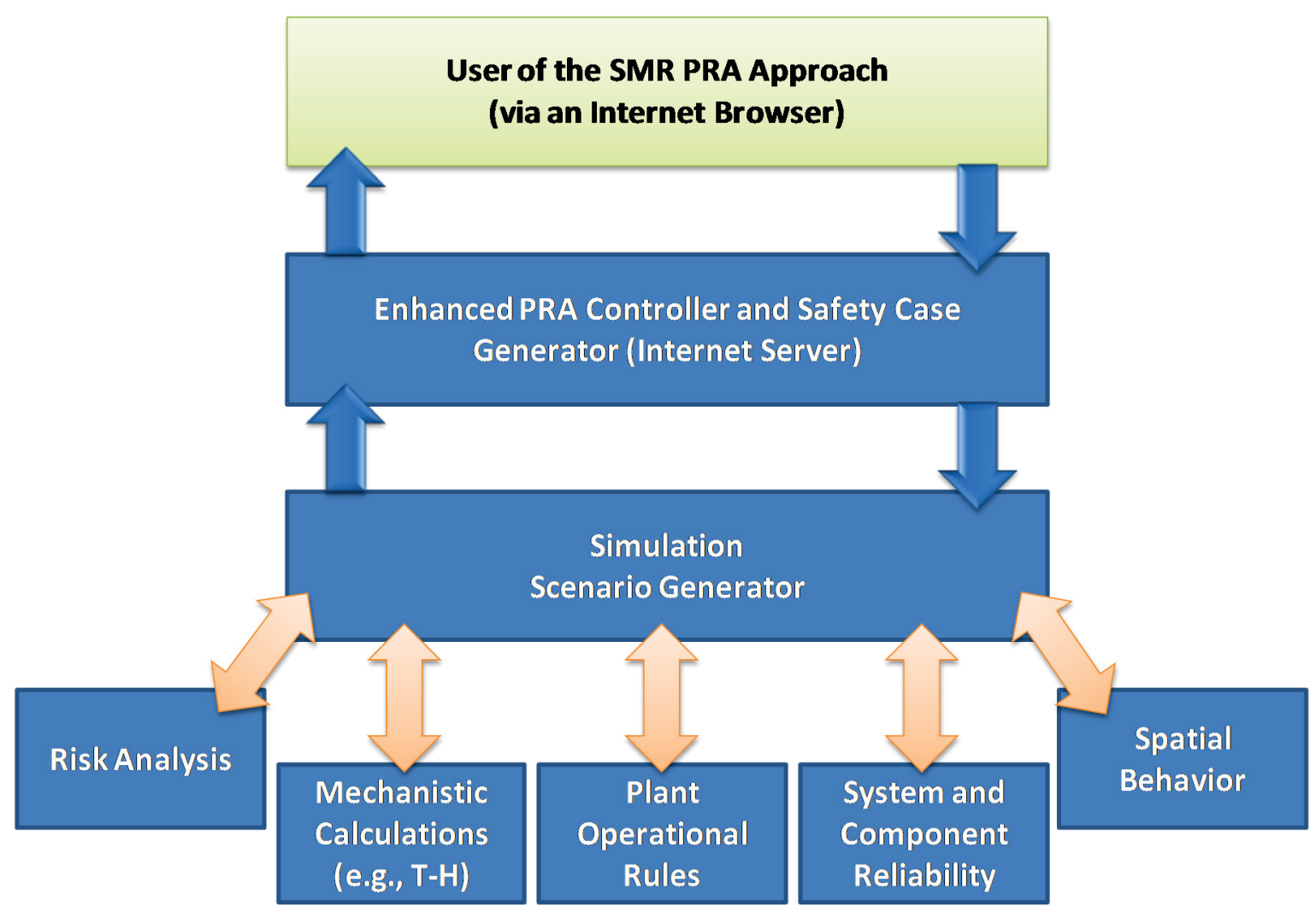

Figure 2. High-level architecture for the SMR advanced PRA framework.

The modules depicted above include:

- An overall PRA controller and module to produce (and store) the safety case. For each performance metric of consideration (e.g., peak clad temperature), this module 
will provide the time- and scenario-dependent results. These results (the "load") will be contrasted to the capacity in order to determine safety margins. Engineering insights will be derived based upon the scenario and associated outcomes (both load and capacity) and are used to document the safety case.

- Simulation for scenario generation. Individual SSCs will be simulated to determine their operability (or not) over time. Coupled to the scenario generator (which is a probabilistic calculation) will be a variety of mechanistic calculations as needed for the scenario.

- Risk analysis including system-level failure models such as simulation, event trees, and fault trees.

- Mechanistic calculations will be used as needed to determine impacts to safety margins. For example, during a seismic event, load and capacity responses to the ground motion may be used to determine component operability.

- Plant operational rules, for example, "rules" including operator procedures, technical specifications, maintenance schedules.

- Knowledge of plant physical properties including SSCs. These models represent component failure models - failure causes and associated information (failures on starting, failures to run, failure rates, etc.).

- Spatial behavior will be used to determine interactions within (and possibly between) the power plant being evaluated. For example, if a fire causes a pipe rupture, the flow of water will be tracked to determine other possible failures in the scenario.

- Uncertainty quantification of both model and parameter sensitivities and uncertainties will be evaluated as part of the overall approach.

One facet of the advanced PRA approach is to find vulnerabilities that affect margins. In general, a margins-analysis approach using simulation-based studies of safety follows the generic process steps:

1. Determine issue-specific, risk-based scenarios and accident timelines.

2. Represent plant operation probabilistically using the scenarios identified in Step 1.

3. Represent plant physics mechanistically based upon the outcome of Step 1 and 2.

4. Construct and quantify probabilistic load and capacity curves related to safety to determine the safety margin.

5. Identify and characterize the factors and controls that determine safety margin within this issue to determine the safety case.

As we simulate these accident scenarios depicted above, we will realize the need to simulate a large number of scenarios. Note that the computational power required for these types of simulations will be able to be shared by many users since the cloud aspect of the PRA tool allows the computations to be centralized. 


\subsection{Physics-based Simulation}

When simulating accident scenarios as part of a safety analysis, we will have the need of several physics-based simulations that must be run for one or more scenarios. A subset of these simulation modules might be run "offline" and their results stored in whatever format is native to that particular application. Alternatively, we may be able to translate these complicated mechanistic calculations into what are called "emulators" wherein the emulator mimics the more complicated analysis but is able to run orders of magnitude faster.

Let us describe a possible PRA scenario to better understand how physics-based simulation is used in the advanced PRA approach.

We construct a model representing the various structures at the SMR. Then, as part of the simulation, we are going to represent a seismic event (which occur stochastically and with different magnitudes) and look at implications to the on-site structures. For a given earthquake that is "produced" by the PRA, we query the results of the structural analysis (which could be a load-capacity calculation using stresses and strains, an emulator-based calculation, or detailed 3D energy transfer modeling) in order to interpret the calculation into a state such as "no damage," "cracked," or "disabled."

The simulation then continues by translating the physics-based mechanistic calculation into an impact in the accident scenario. For example, if the structure is cracked, this state would be applied to the component in the model (perhaps it is a wall or a pipe) using another stochastic model (in this case, a cracking model). Once the component state is specified, then the scenario would continue since the cracked component may experience a dislocation (the crack grows) or further damage. If the component is a pipe containing water, then we might experience a flow out of the pipe at the point of the crack, which could be in a critical location in the SMR. The water outflow from the pipe would immediately result in two impacts:

- Reduced flow past the crack (possibly reducing heat transfer at the point where the water was originally needed)

- Possible spatially-related damage, depending on the location of the leaking water, and near-by components.

While the spatial interactions are being represented in the 3D environment, the accident scenario generator continues since water flowing from the leak may (later in time) fail collateral components (say a pump in the same room as the leaking pipe). At this point in the scenario, we are representing a flooding scenario (that was initiated by a seismic event). Further, there may be other components in that room that are sensitive to the leaking water, for example the pump motor controller which is an electronic component.

Note that this example scenario described above is just one possible outcome of the seismic event. However, it is the coupling of probabilistic and mechanistic calculations together that will, in the advanced SMR PRA, be able to search (automatically) for vulnerabilities. Further note that a variety of special-type scenarios may be modeled and represented in these advanced models, including fire propagation, physical damage, flooding, and seismic 
impacts. A variety of 3D physics toolkits are available, both commercially and through open source, and typically have features including:

- Collision detection (to know when something hits something else)

- Solvers for rigid body dynamics (to mimic realistic movements)

- Fluid, particle, and character controllers (to represent fluids, movement of objects, and representation of people)

- Articulated mechanical dynamics (to represent complex components and systems)

- Fluids allowing the simulation of liquids and gases using a particle system and emitters.

\subsection{Example use of 3-D Physics Toolkits for PRA}

To better understand how 3D environments can assist in risk assessment, we provide an example. We will simulate hazards such as an earthquake. For example, during a simulation run, assume we see an earthquake event at a time of 22.3 years.

Upon the arrival of the earthquake (at year 22.3 in the virtual facility), the power may be failed at the external pumping station for the facility. In this example, we assume that a portable diesel generator has been brought to the site and setup for the pumping station. As water fills the pool and the station operates (see Figure 3), a pipe facture caused by the earthquake causes water to spray out and hit the generator (see Figure 4). This water damage is detected by the simulation and reported to the PRA model. Note that this entire scenario is produced and managed "on the fly" by the simulation 3D environment - the scenario is not scripted a priori (unlike an event tree model where the scenario is described, generally at a higher level, by the analyst). Also note that the next simulation case for a similar type of earthquake will (most likely) produce a different set of outcomes. By running a large number of simulations, we can understand the behavior of the SMR facility and look for potential vulnerabilities. Note that the pictures shown in Figure 3 and 4 are snapshots of single points in time from an entire scenario (which was captured as a video, and can be shown to analysts for further consideration and understanding). 


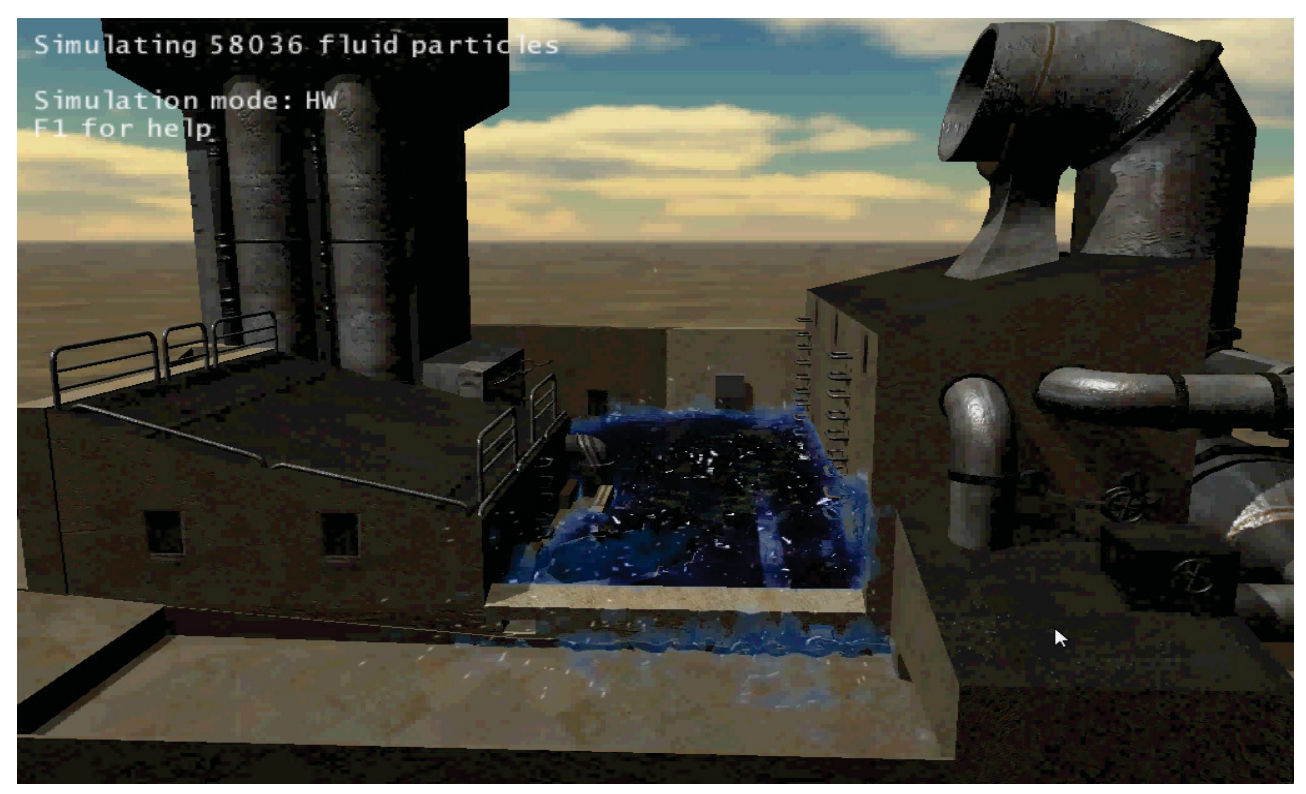

Figure 3. Pumping station pool simulation showing the water physics.

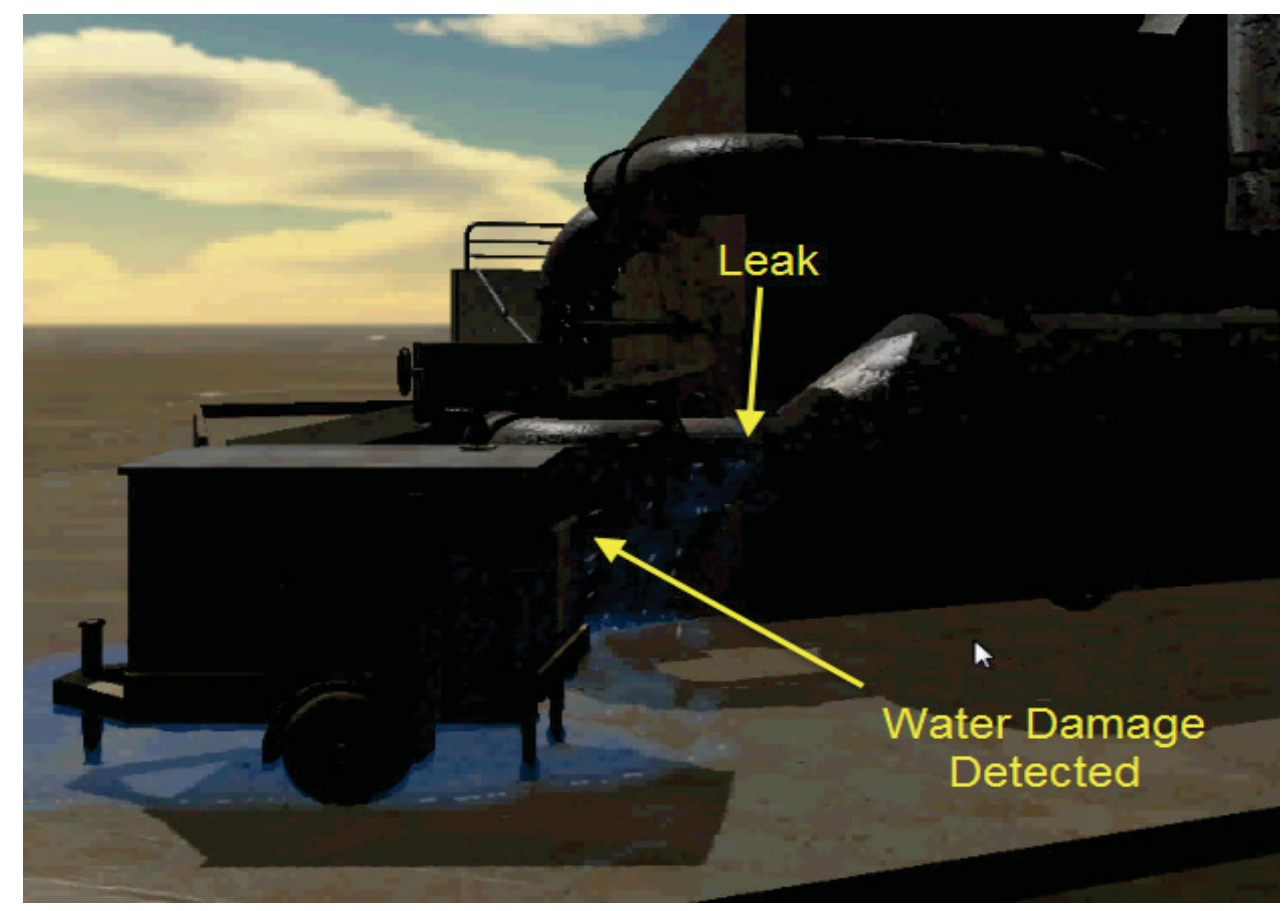

Figure 4. Leak and water damage detection by the simulation physics.

Through the advancement of 3D modeling and simulation, complex interactions between objects in advanced models can automatically be determined through calculated physics in real-time. These advancements give us the opportunity to rethink how we approach PRA. Instead of analysts having to think of both accident scenarios and the possible outcomes; they would only have to come up with the scenarios and then "see" the outcomes. 


\subsection{PRA Interaction}

Software or code can interact or receive information from a physics engine simulation (for example, to trigger a condition when a single drop of water hits a critical component).

Through this capability, PRA could know the occurrence of various events in the simulation. This is done through sensors or triggers placed in the model. For example, a trigger can be placed on an item for a collision, so if an item collides with it that trigger is activated (see Figure 5). The trigger activation sends a notification to the PRA software so that it can take appropriate action (e.g., fail a component that may impact other systems or trigger an alarm in a control room).

An item in the 3D model may represent a component of the PRA model, but a sensor or trigger may be attached to one or more basic events of the model. Also those sensors may trigger different basic events depending on the time of event. For example, sensing water on an electric valve may trigger (logically set the event to "true") the valve's basic event of 'fails to open' if the valve is currently closed, or it may trigger the 'fails to close' basic event if it is open at the time of the trigger.

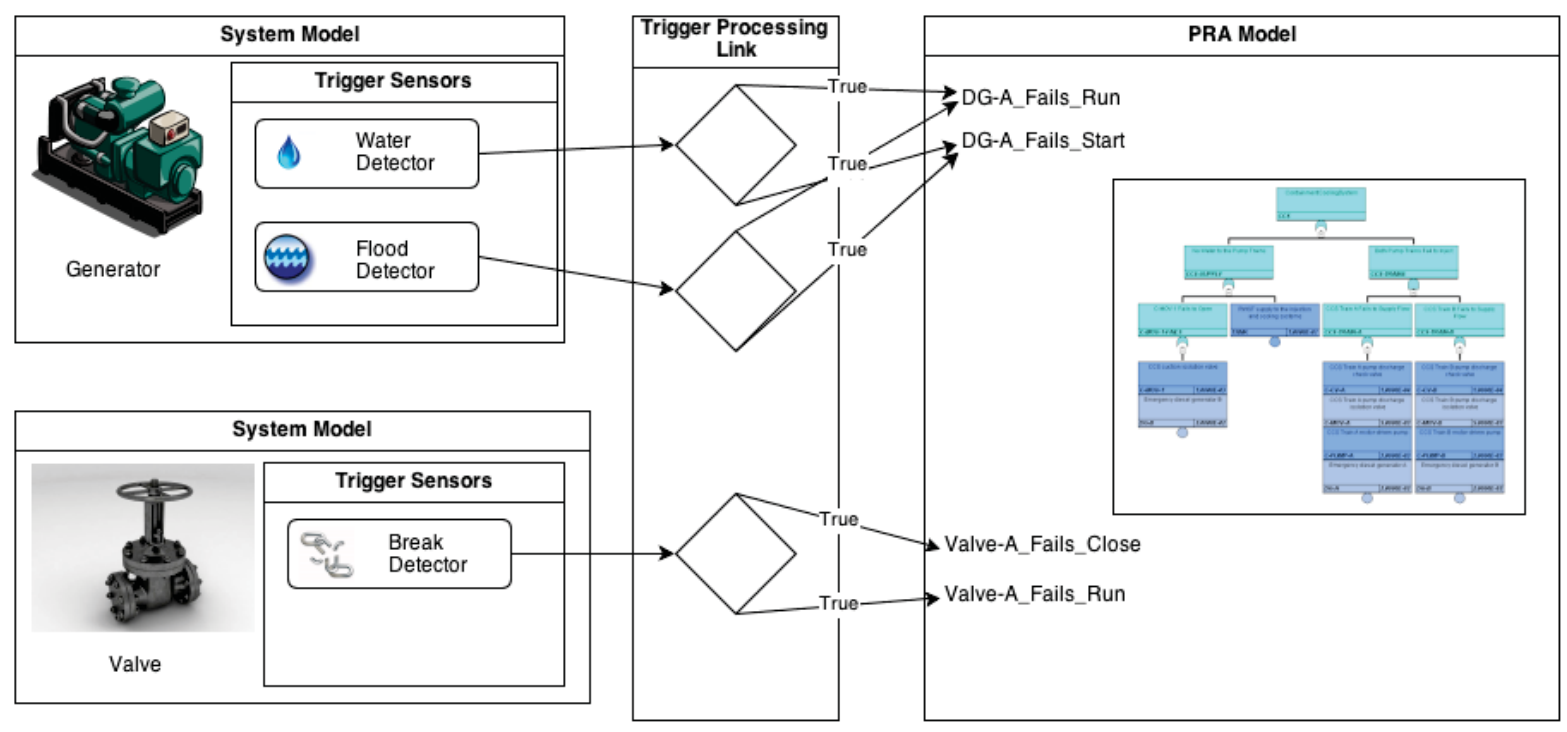

Figure 5. Correlation between assigned model trigger sensors and PRA Basic Events

By running the simulation with these time dependent interactions, an analyst could see not only what impacts these events have on the plant, but the relative time relationship between the events. 


\section{Chapter \\ Analysis Results and Insights}

The analysis described in this report presents an evolution of how risk assessment for hazards such as external events can be treated. In general, external events analysis is performed following the approach shown in Figure 6. However, in our assessment, we will be demonstrating analysis by using advanced 3D facility models coupled with simulation in order to represent flooding hazards that could be present at a hypothetical SMR facility.

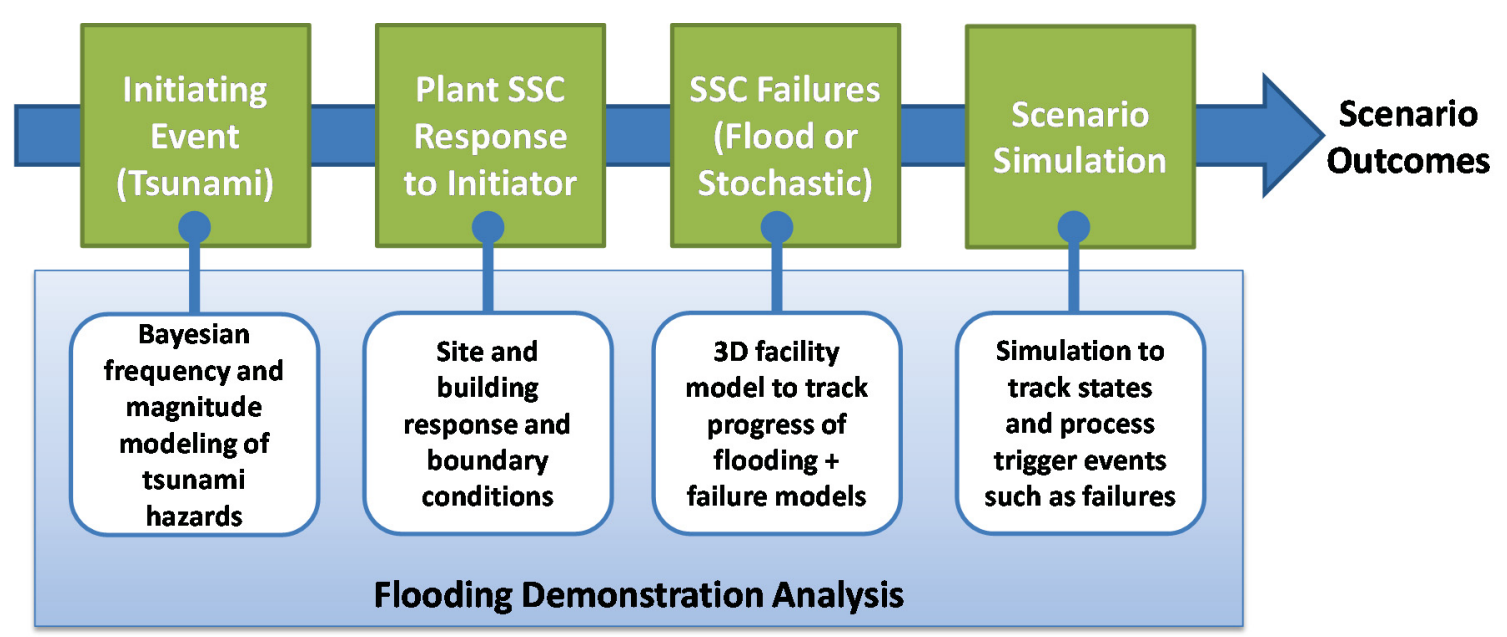

Figure 6. Representation of the simulation process for external events.

\subsection{Bayesian Modeling for Extreme Event Initiators}

The first step in the analysis is to perform Bayesian analysis of initiating events, including the frequency of tsunami events. The goal of the Bayesian modeling for extreme event initiators (e.g., tsunamis) is to gain insight as to the expected tsunami height for various return periods given historical data, and to make predictions as to the expected tsunami heights for longer return periods at a given plant location. The desired result is a distribution of expected tsunami heights for various return periods (i.e., yearly, every 10 years, every 100 years, etc.) based upon observed historical events.

Historical tsunami data for this study was obtained from the National Oceanic and Atmospheric Administration (NOAA). The data is available for download from the NOAA website, http://www.ngdc.noaa.gov/hazard/tsu_db.shtml. The download file contains details for all known tsunamis world-wide from 2000 B.C. to present, including; maximum wave height, magnitude and location of seismic initiating event, and other attributes. The 
validity and accuracy to be expected from historical events are also graded to allow for scrutiny and segregation of the data, if desired.

The case analyzed and discussed in this section includes all known historical tsunami events affecting the Fukushima Prefecture region of Japan. For the case study described in this report, we assume that our hypothetical nuclear power plant will be located on the north-east coast of Japan, in the Fukushima Prefecture region. In order to obtain these data from the large data file from NOAA, all tsunamis affecting Japan were retained from the data file. The data were then segregated by the location of the seismic event that generated the wave. Any data that initiated within appropriate latitude and longitude that the Fukushima Prefecture could be impacted was retained for analysis and the other data were discarded. The latitude and longitude used for parsing the data was 35 - 40 degrees north and 141 - 144.5 degrees east, respectively. This resulted in data ranging from the years 1611 to 2013 (a span of 403 years). A sample of the data retained is illustrated in Table 1.

Table 1. NOAA Tsunami Data (example).

\begin{tabular}{|cccccccc|}
\hline YEAR & MONTH & DAY & $\begin{array}{c}\text { EVENT } \\
\text { VALIDITY }\end{array}$ & $\begin{array}{c}\text { FOCAL } \\
\text { DEPTH }\end{array}$ & $\begin{array}{c}\text { PRIMARY } \\
\text { MAGNITUDE }\end{array}$ & LATITUDE & LONGITUDE \\
1938 & 11 & 13 & 4 & 60 & 6 & 36.7 & 142.1 \\
1938 & 11 & 6 & 4 & 20 & 7.1 & 37 & 142.2 \\
1938 & 11 & 30 & 4 & 50 & 7 & 37.5 & 142.2 \\
1938 & 11 & 5 & 4 & 30 & 7.7 & 38.2 & 142.2 \\
1938 & 11 & 5 & 4 & 30 & 7.6 & 37.552 & 142.214 \\
1938 & 11 & 6 & 4 & 0 & 7.5 & 36 & 142.3 \\
1959 & 1 & 22 & 4 & 40 & 6.8 & 38.297 & 142.373 \\
1959 & 10 & 26 & 4 & 10 & 6.7 & 38.1 & 142.4 \\
1960 & 3 & 23 & 4 & 20 & 6.7 & 39.5 & 142.5 \\
1960 & 3 & 20 & 4 & 20 & 7.5 & 37.812 & 142.619 \\
1961 & 1 & 16 & 4 & 20 & 6.4 & 38.64 & 142.75 \\
\hline
\end{tabular}

Bayesian Inference was performed using these data to determine the expected maximum wave height for the one year, 10 year, 100 year, and 1000 year return periods. Several distribution types were used in this Bayesian Inference, including: gamma, exponential, and lognormal. The gamma and lognormal distributions did not produce a sensible distributions for the 1000 year return period and were then rejected from further analysis. The exponential distribution yielded viable results for all four return periods, with increasing uncertainty as the return period grew, as expected. The distributions used for each wave height for the four return periods are given in Table 2 , along with their associated $5^{\text {th }}, 50^{\text {th }}, 95^{\text {th }}$ percentiles and mean values. The cumulative distribution function (CDF) for the four return periods are given in Figure 7, and wave height for each return period are illustrated in Figure 8. 
Table 2. Wave Height Distributions for Each Return Period.

1 year return period: exponential distribution with $\lambda_{\text {mean }}=1.90 \pm 0.095$

$5^{\text {th }}=0.03 \mathrm{~m} \quad 50^{\text {th }}=0.36 \mathrm{~m} \quad$ Mean $=0.53 \mathrm{~m} \quad 95^{\text {th }}=1.57 \mathrm{~m}$

10 year return period: exponential distribution with $\lambda_{\text {mean }}=0.249 \pm 0.039$

$5^{\text {th }}=0.21 \mathrm{~m} \quad 50^{\text {th }}=2.79 \mathrm{~m} \quad$ Mean $=4.02 \mathrm{~m} \quad 95^{\text {th }}=12.06 \mathrm{~m}$

100 year return period: exponential distribution with $\lambda_{\text {mean }}=0.044 \pm 0.020$

$5^{\text {th }}=1.18 \mathrm{~m} \quad 50^{\text {th }}=15.9 \mathrm{~m} \quad$ Mean $=22.9 \mathrm{~m} \quad 95^{\text {th }}=68.7 \mathrm{~m}$

1000 year return period: exponential distribution with $\lambda_{\text {mean }}=0.026 \pm 0.025$

$5^{\text {th }}=1.99 \mathrm{~m} \quad 50^{\text {th }}=26.9 \mathrm{~m} \quad$ Mean $=38.8 \mathrm{~m} \quad 95^{\text {th }}=116 \mathrm{~m}$

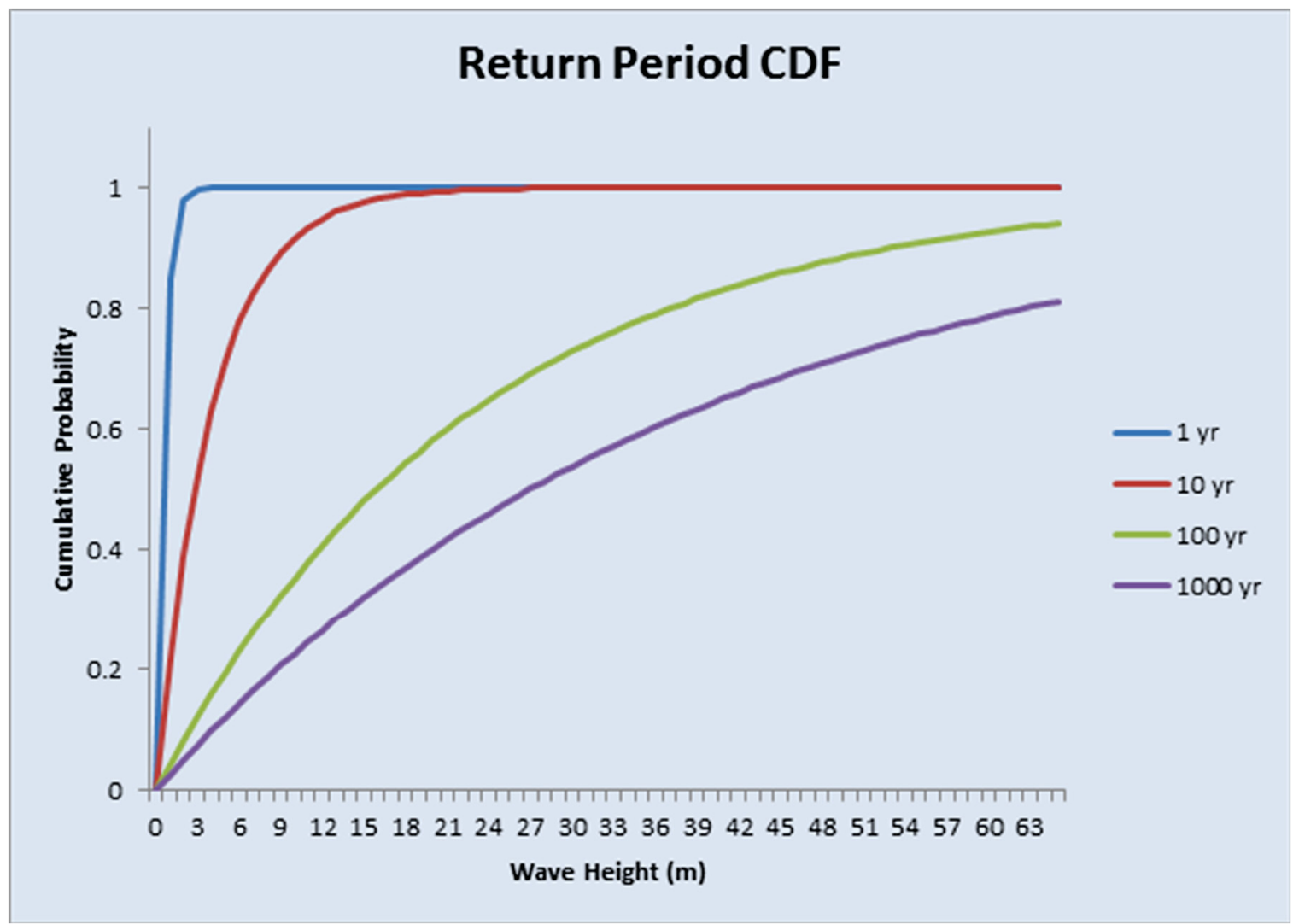

Figure 7. Return Period Cumulative Distribution Functions. 


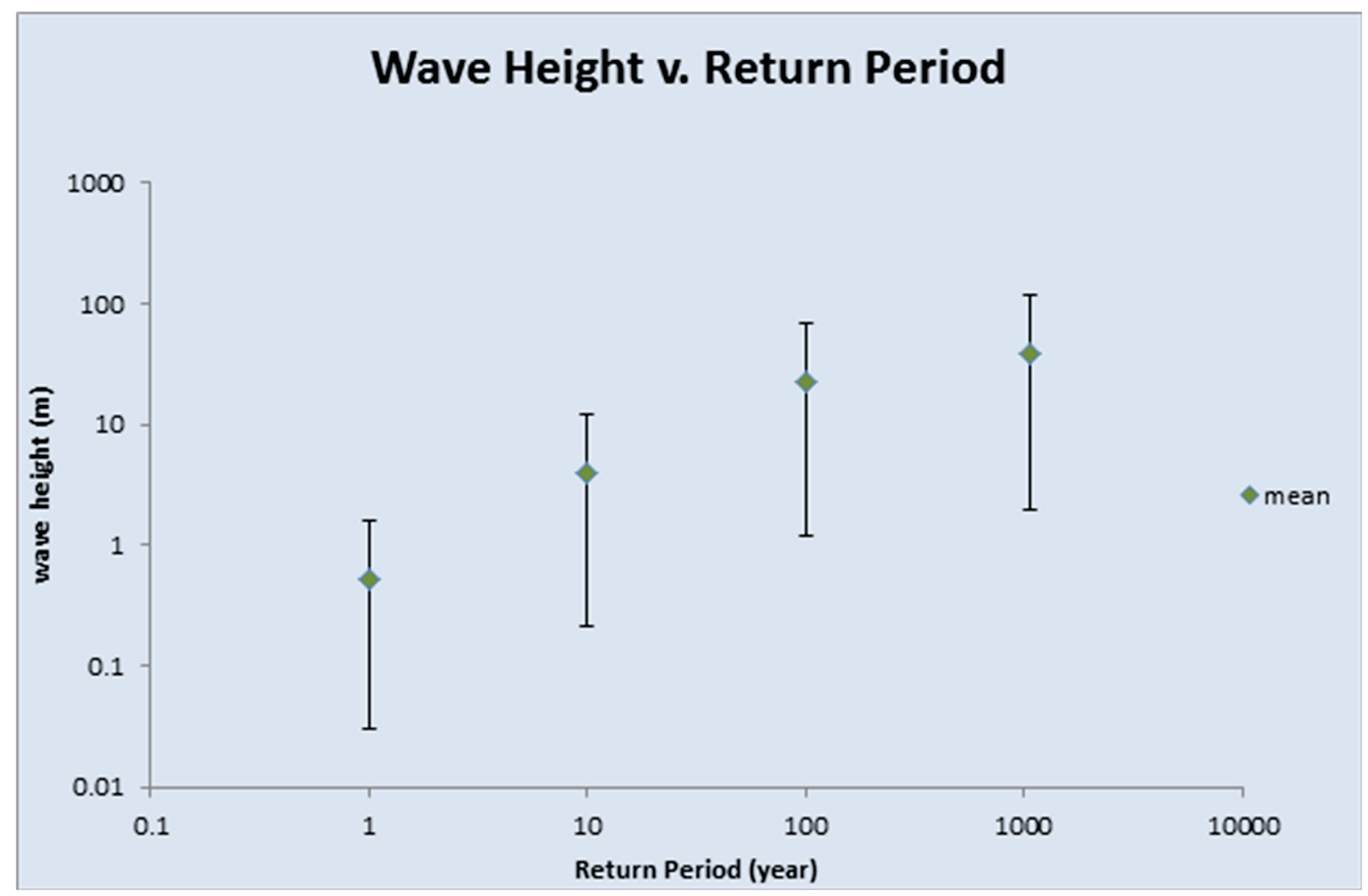

Figure 8. Wave Height for Various Return Periods (based on historical data).

The historical data was sufficient in developing wave heights for observed return periods, but unable to predict maximum wave heights to be expected for longer return periods. An "extreme value paradigm" is used in order to extrapolate observed data for estimation of the predicted return period wave heights for longer periods (i.e., 10,000 years and 100,000 years). Specifically, the generalized extreme value (GEV) family of distributions is used to perform an asymptotic extrapolation of the known data. For this hypothetical example, we created an OpenBUGS script to perform the Bayesian inference, building upon the Generalize Extreme Value (GEV) modeling as described in [2]. Additional details on the GEV model and assumptions may be found in Chapter 13 of [2]. The OpenBUGS script that was produced is shown below.

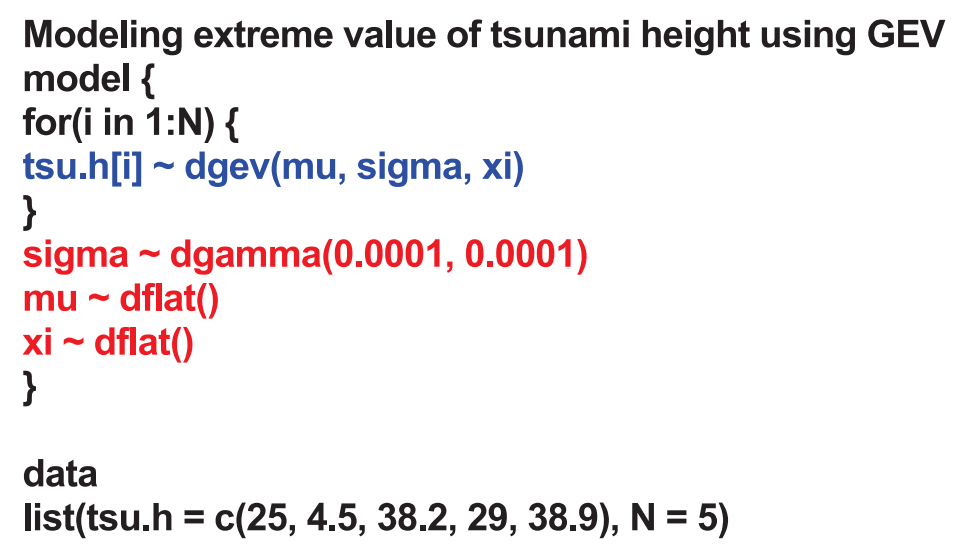


The GEV distribution is given below and uses three parameters; mu, sigma, and xi. Mu can be thought of as a location parameter, sigma is a scale parameter, and $x i$ is the shape parameter of the distribution.

$$
G E V(z)=\exp \left\{-\left[1+\xi \frac{(z-\mu)}{\sigma}\right]^{-\frac{1}{\xi}}\right\}
$$

When $\mathrm{xi}>0$ the asymptotic extrapolation approaches a maximum return period, but there is no upper bound on the predicted maximum wave height; $x i<0$ the asymptotic extrapolation produces a maximum predicted wave height with no maximum return period.

The first attempt to employ GEV Bayesian Inference was with the annual maximum tsunami height for years 1611 to 2013. Not every year had a recorded tsunami and some years had two or more small tsunamis. Since seismic events along faults happen every day, given they are small in magnitude, each year that did not have a recorded tsunami was recorded as 0.1 $\mathrm{m}(10 \mathrm{~cm})$, which is well below high tide and any measurable wave height. The maximum tsunami height within a year was taken for all years that had more than one recorded tsunami. This produced 403 data points for the inference analysis. The posterior parameters were; $\mu=0.2426, \sigma=0.5033$, and $\xi=3.497$. This resulted in predictive wave heights of 3.76 $\mathrm{m}, 1.4 \mathrm{E}+6 \mathrm{~m}$, and $4.5 \mathrm{E}+9 \mathrm{~m}$ for the 10 year, 100 year, and 1000 year return periods, respectively. These results do not appear to be representative of historical observations and are a result of the relatively large xi value.

The second attempt to perform the GEV Bayesian Inference used the maximum wave height for ten year periods (instead of every year). There were some decades in which no recorded tsunami occurred and others in which several tsunamis were recorded. The same approach as above was used to adjust the data as for the annual return period. The posterior parameters using the 10 year return period data were; $\mu=1.239, \sigma=5.293$, and $\xi=4.649$. Once again, xi was very positive resulting in no upper bound for the predicted maximum tsunami wave height. The main reason the 10 year and one year return period data results in a xi greater than zero is because of numerous years in which no tsunami is recorded or only a small tsunami occurs. This causes an artificial growth in predicted wave height when years with large tsunamis are added to the data base.

The third attempt to perform the GEV Bayesian Inference method was as before except using data for the maximum wave recorded for each 100 year period. This resulted in no zero values used in the analysis since all the data ranged between $4.5-38.9 \mathrm{~m}$. The posterior parameters here were; $\mu=23.3, \sigma=29.58$, and $\xi=-1.645$. The 100 year return period data resulted in a negative value for xi which results in an asymptotic extrapolation from observed data to larger return periods. These parameters predict a mean value of the maximum possible tsunami height for any return period beyond 100 years to be less than or equal to $41 \mathrm{~m}$. Figure 9 gives the predictive wave heights for larger return periods along with the previous results from above. 


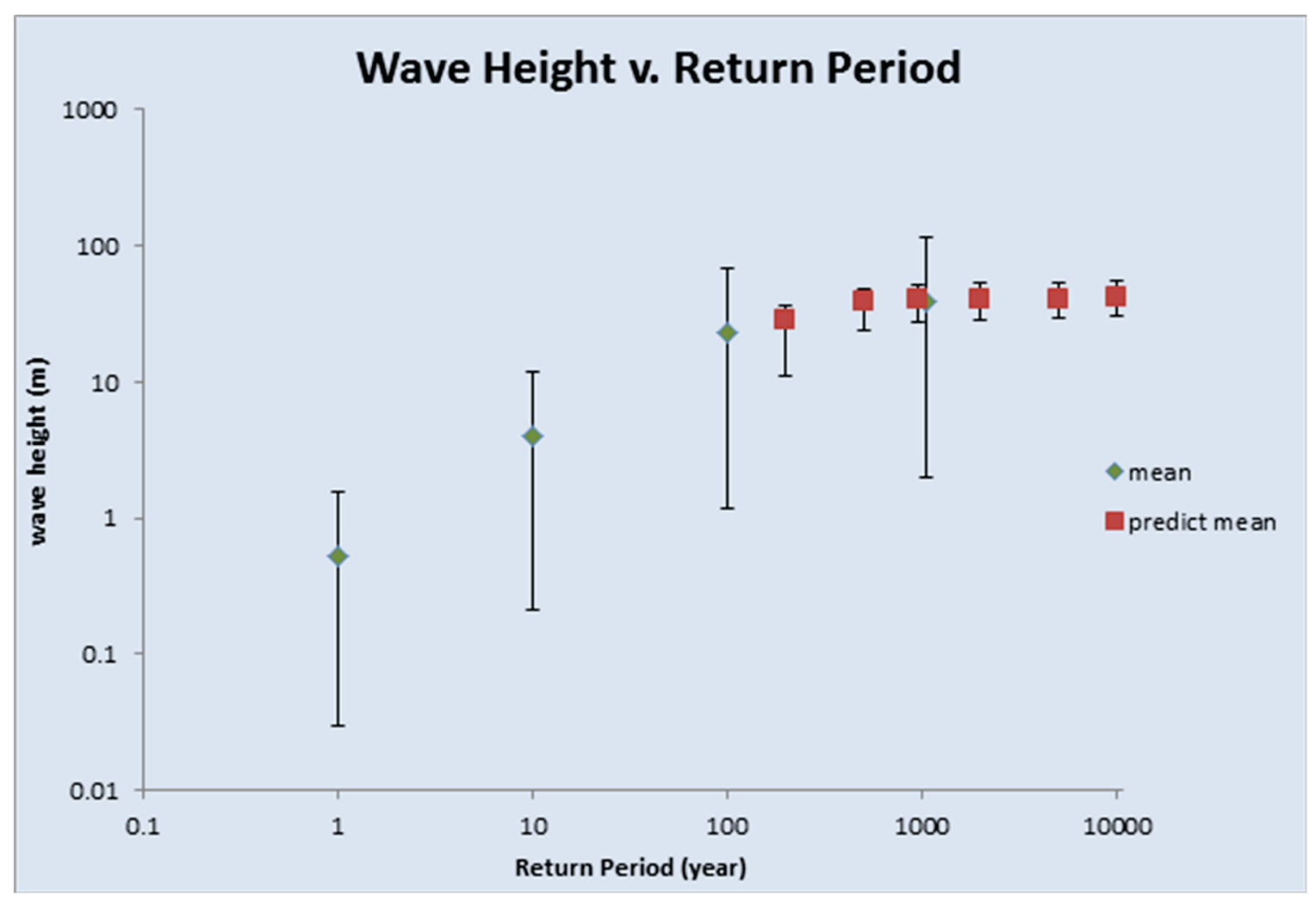

Figure 9. Wave Height for Various Return Periods

\subsection{SSC Failure Modeling}

\subsubsection{State Modeling}

To run a simulation, a model of the system must be constructed based on how it behaves in time. This modeling is done using an event and action driven state diagram approach. At any given moment the model is in a set of "States". Each state can have "Actions" it performs upon entering that state and "Events" that trigger an action or set of actions. The set of "Current States" changes over time until a terminal state is reached. Once a terminal state is reached, the "Current States" list is evaluated and logged as one iteration of the simulation (the black dot in Figure 10). 


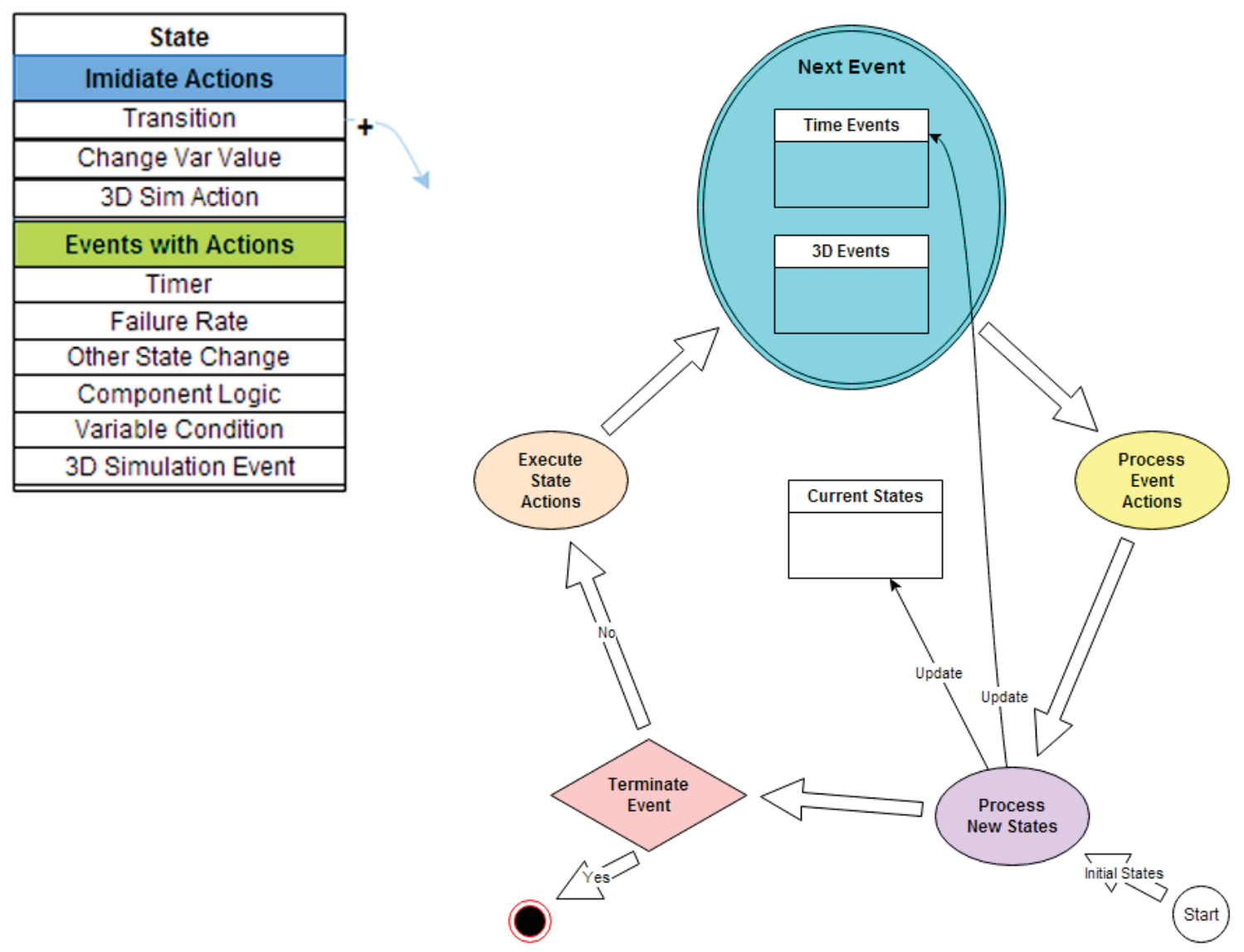

Figure 10. State Action and Event options (Left), flow of State processing (Right)

\section{Definitions}

State: a logical representation for the condition of a component or system. (4 types)

1. Start - A state that is to be placed in the current state list when the model begins a simulation.

2. Standard - A normal state representing no special conditions.

3. Key State - Marks a state that is to be tracked for final probability calculations. (All "End States" from a traditional PRA model should have a corresponding "Key State")

4. Terminal - Marks when a simulation ends. (If this state is encountered then the simulation ends)

Component Group: a group of states that together define the valid states of a component. Only one of these states can be in the "Current States" list at any given time. Each of these states must have a success or failed flag indicating if the component is in an "OK" or "Failed" condition.

Variables: named values that can be set by "Actions" or evaluated by "Events". (3 Types)

1. 3D Simulation - value for the associated component in the 3D simulation.

2. Component - available for all to read but only "Actions" in a "State" associated with that component can change the value.

3. Global - available for all to read the value and "Actions" to set it. 
Action: (3 types).

1. Transition - Start or move to a new state or states. It is probabilistic if it contains more than one to state.

2. Change Value - Change the value of a variable.

3. 3D Sim Action - Send a message to the 3D simulator.

Event: A condition based item that when met executes its assigned actions. (6 Types)

1. Timer - executes when time has passed.

2. Fail Rate - executes when the sampled time (based on the failure rate) has passed.

3. State Change - executes when the associated state is in the list of current states.

4. Component Logic - executes if the defined logic for a set of components is met. (Similar to evaluating a FT in PRA without probabilities)

5. Variable Condition - executes if a variable meets the user defined condition.

6. 3D Simulation - executes if the associated 3D component fails.

The state diagrams can be defined based on PRA modeling practices of components with various failure types, fault trees, and event trees. For simplicity, most of the following examples and diagrams will be describing the "Advanced Demo" project in SAPHIRE (which is equivalent to the example used in [3]).

Each component has various states that it can be in such as Standby/Off, Active/On, and Failed. The paths from one state to another are dictated by events. For example, a failure type is handled through an event. A failure to start would use an event placed on the standby state. When a start system request is made, it either moves to the "On" state or the "Failed" state determined by the probability associated with it (see the red circled event in "E-PUMPB_Standby" of Figure 11). A failure to operate/run would be represented with an event in the active/on state going to the failed state, the event produces a time based reaction by sampling the probability associated with it (see the red circled event in "TANK_Active" of Figure 11). 

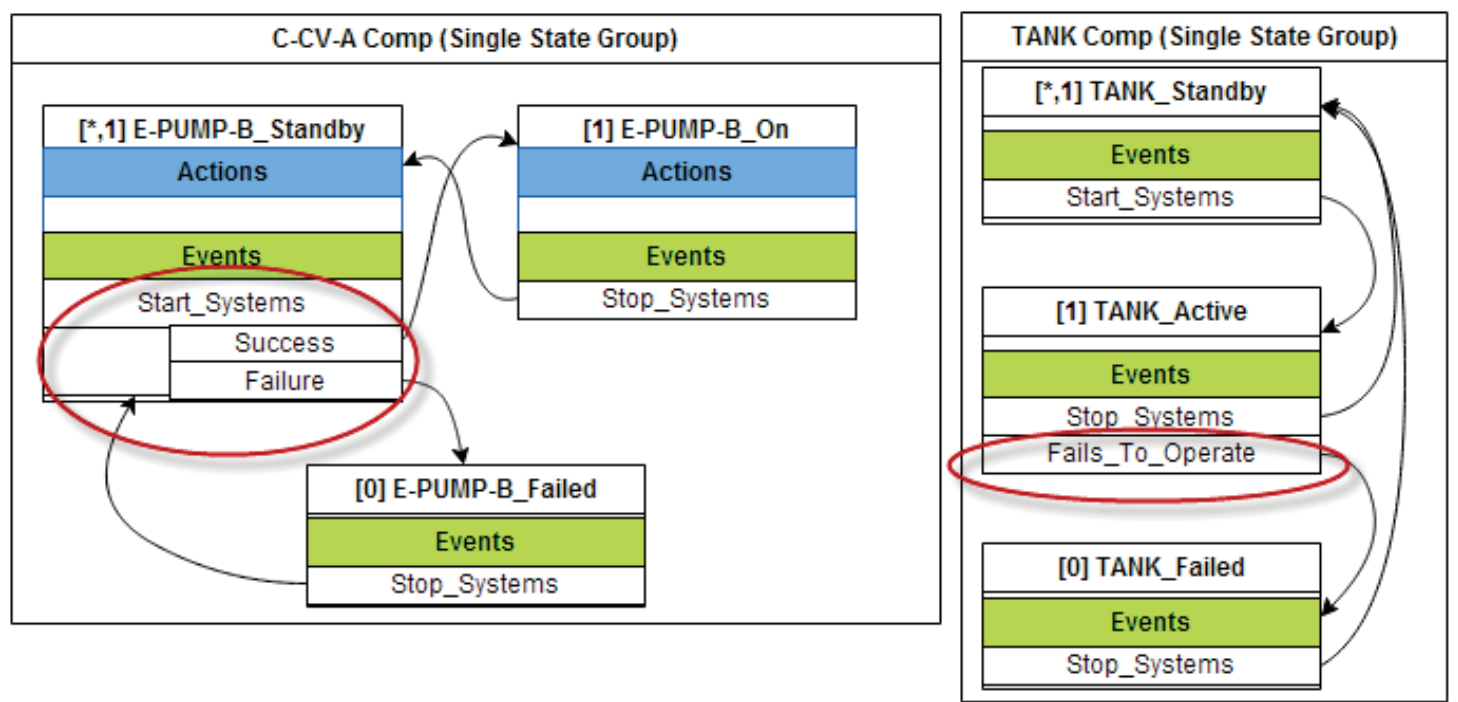

Figure 11 - Example for state transitioning for components and types of failures. (Left) Fails to Start (Right) Fails to operate or run.

If the component has both failure types then it is just a combination of the two events in the corresponding states (as shown by E-PUMP-B in Figure 12).

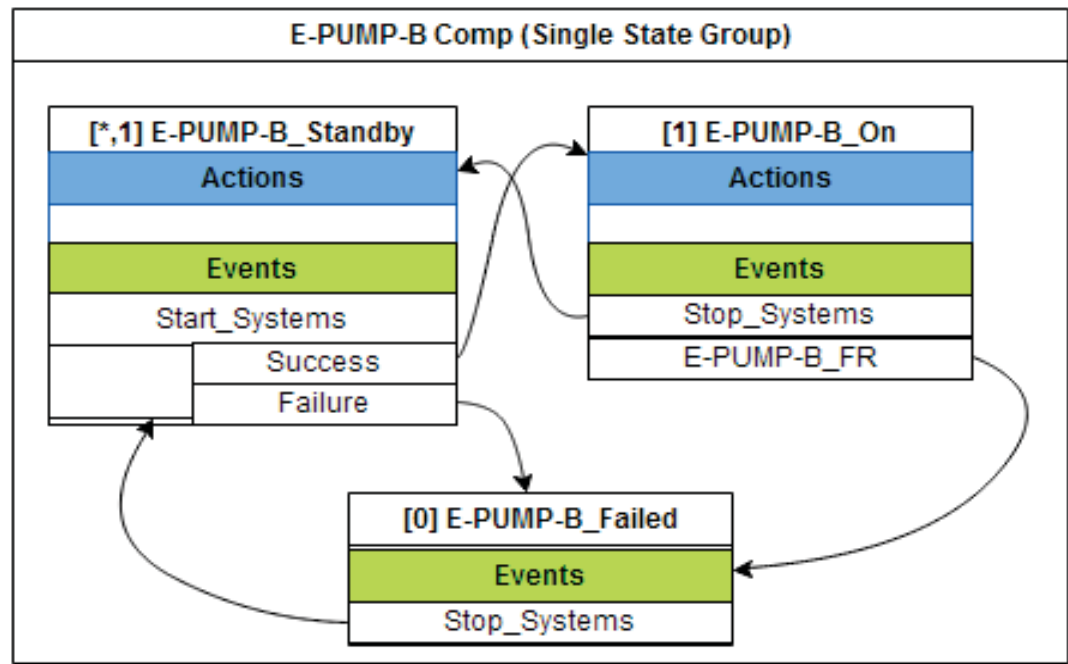

Figure 12 - Example of State flow for a component that has both "fails to start" and "fails to run".

When adding states for a component, each state is flagged as either a success or failed state for the component (designated by the [0] or [1] before the state name, see Figure 13). This flag allows for an evaluation of the component by other events in the model.

Once all component states have been modeled, systems can be represented using component logic events. A system failure event uses a logic diagram of the components and evaluates its success or failure to trigger the event's actions (see Figure 13). This is similar to a typical PRA model except this is based upon simulation at a component level. 
The states for the fault tree system could be as simple as an active and failed state with movement between them triggered by the previously described event (see Figure 13).
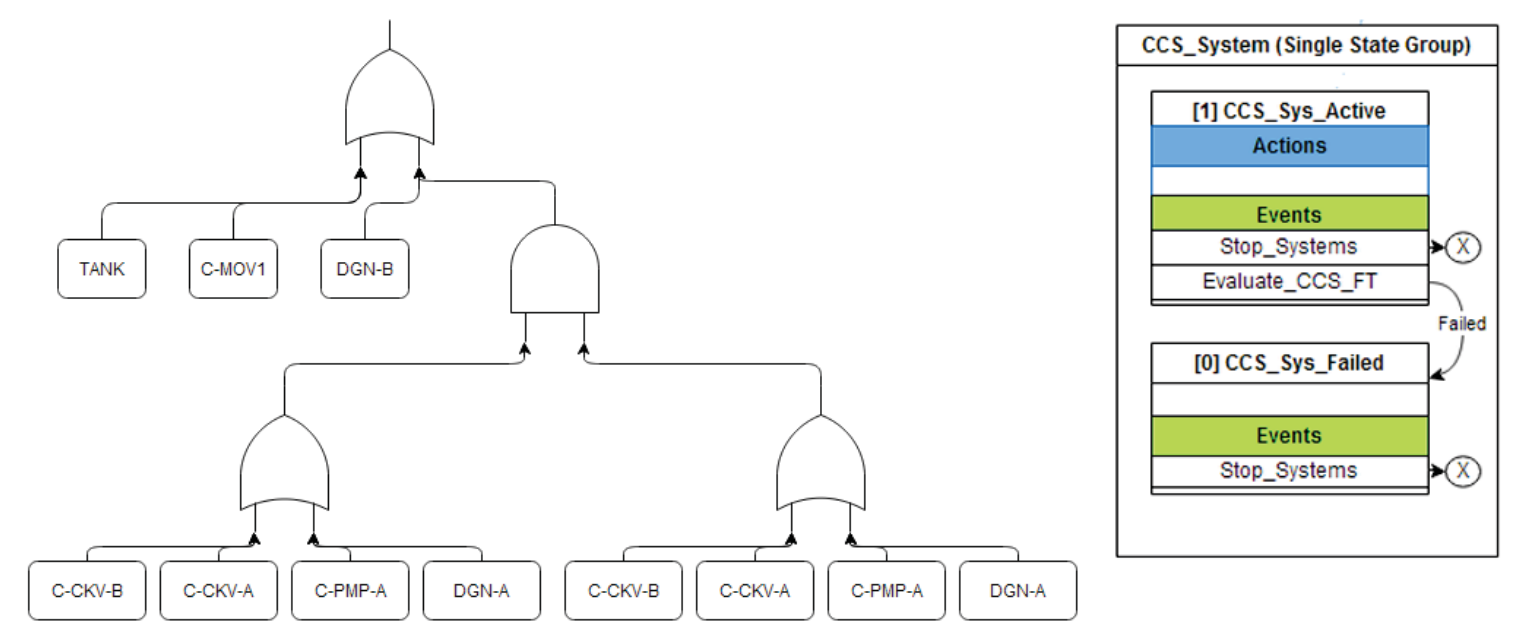

Figure 13 - Example of Logic Evaluation for triggering an event

With all the systems modeled, the next thing is to track the critical or end states of the plant. This is done by starting with a normal operations state with initiating events transitioning to other plant states and those leading to desired end states. It also needs a terminal state to end the simulation with a mission-time state and other final states transitioning to this terminal state.

In the Advanced Demo example we have the following states (see Figure 14).

Normal_Op - the starting state for the simulation, with the initiating event IE-LOSP transitioning to the LOSP state. It also has an immediate action of going to Stop_Systems in order to reset everything if it goes back into normal operations. ("+" indicates adding a new state instead of leaving this one)

LOSP - Indicates that the system is without off-site power. When entering this state, we need to immediately activate all the systems of the plant and monitor events that constitute a small or large release.

Stop_Systems - A temporary state use to turn off the evaluation of any component and systems. (The empty action with the arrow to a circled " $X$ " indicates an immediate exit of the State.)

Start_Systems - A temporary state used to trigger any failures for components and to evaluate systems.

Small_Release - An end state that results are needed for the overall analysis. The only way to leave this state is to have a large release which supersedes the small release. 
Large_Release - An end state that results are needed for the overall analysis. This state also triggers an end of the simulation.

MissionTime - A starting state used to shut down the simulation after the mission time has elapsed.

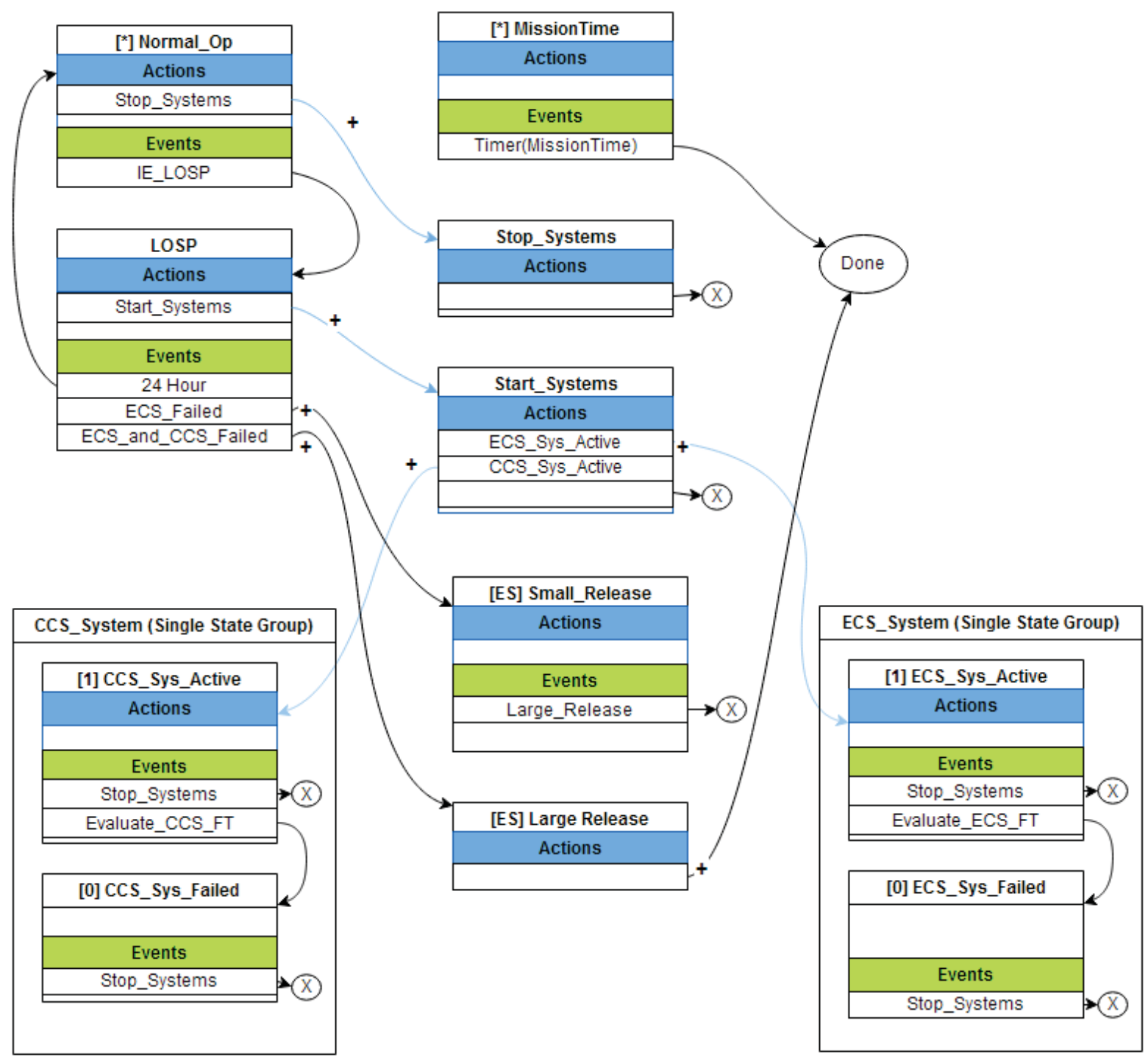

Figure 14 - State diagram example for evaluating Systems and tracking End States of the "Advanced Demo" project in SAPHRIE.

\subsubsection{D Modeling}

In order to capture failures caused by physical interactions, a 3D physics simulation is needed. This is done by first constructing a 3D model of the desired facility (for this example, we used publically available models that represent generic nuclear facilities). Next, we create 
the incident or scenario we wish to consider. All components that can be affected by the scenario must have been modeled and associated with the events we are concerned about, including those such as water contact, flooding, or impact from other components (see circled items in Figure 15).

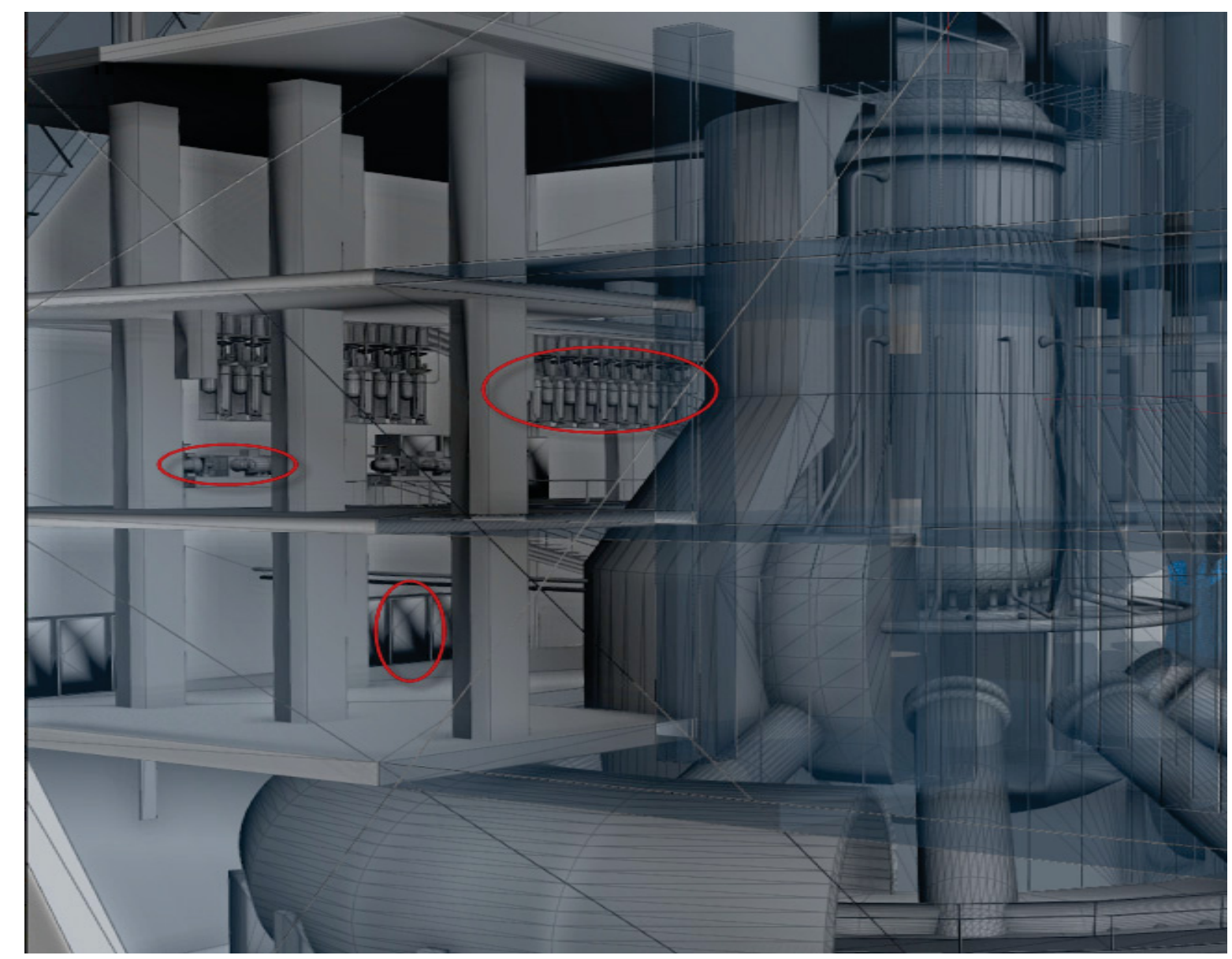

Figure 15 - Components in a 3D model.

For our 3D simulation and notifications, a software package called Houdini FX was used. This application is a dynamic and widely used 3D simulation environment that allows for custom modification to every important aspect of the simulation. This customization allowed us to develop a plug-in for a communication protocol with our state diagram simulator, thereby interfacing the probabilistic simulation with the mechanistic 3D flooding analysis. With this synchronization between the two simulators, we can incorporate events or results from the two different methods to produce a more accurate overall analysis.

\subsection{Scenario Simulation}

By using the SAPHIRE model, we can perform a comparison between the simulation model and the static model in order to establish a baseline before adding other features or states 
such as a 3D mechanistic simulation. The state simulation for the Advanced Demo project, described previously, was run 1,000,000 times with Small_Release and Large_Release end states being tracked. The results were compared to the results from the demo model in SAPHIRE (See the results in Section 2.4). With insignificant differences in the results, it can be concluded that the simulation is an accurate baseline for comparison.

For our testing, we were initially focusing on the flooding caused in one specific area of the facility. In order to get flood water flow data, we created a 3D tsunami simulation with various heights of waves (see Figure 16 for a representation). The water height from these simulations were converted into a flow rate over time for a single room 3D simulation.
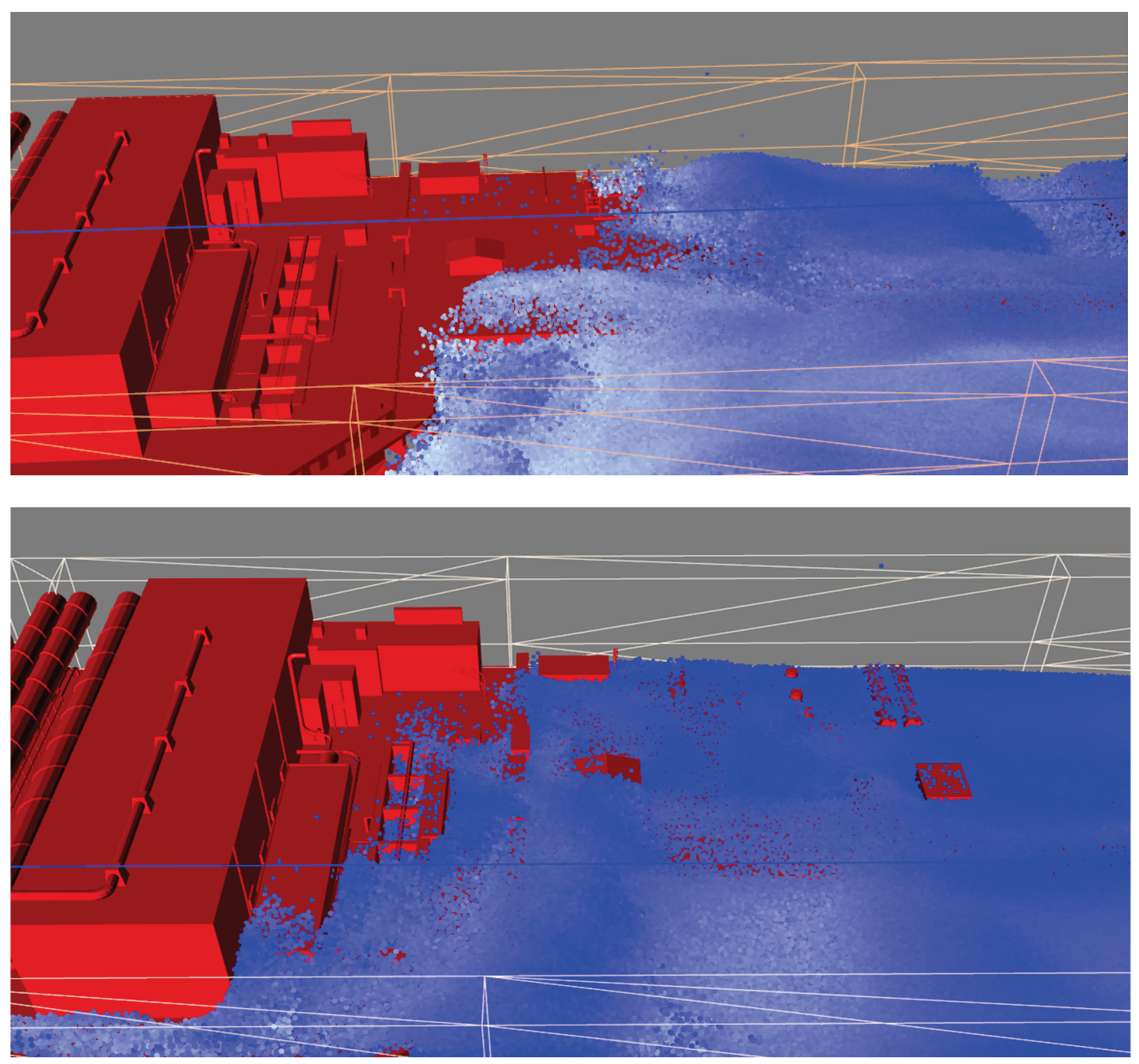

Figure 16 - 3D flooding simulation.

Adding the 3D physics simulation requires just a few steps. First, we have a 3D scenario to run with the desired components tracked. Second, these components must also have a corresponding 3D event in the state diagram to indicate what to do when information from the 3D simulation is received, for example, if a component is flooded (see the circled event in 
Figure 17). Finally, an action to start and to stop the 3D simulation must be added at the desired point in the main flow section of the state diagram.

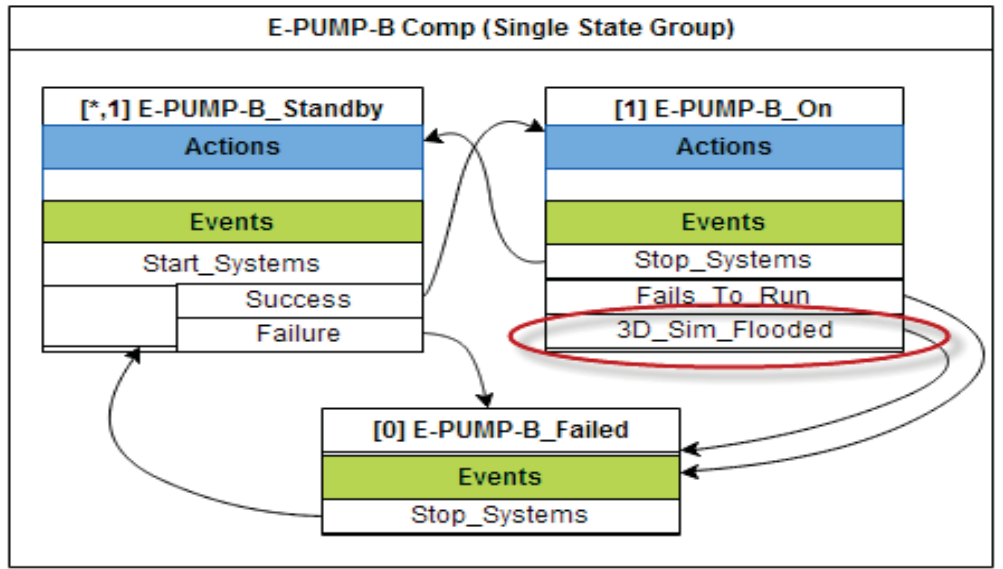

Figure 17 - Example of adding a 3D event to move to a failed state.

For the Advanced Demo example, we wish to know how flooding of a room from varying tsunamis would affect the overall results. To test this approach, we constructed a room 3D environment where the water ingress could affect critical components (pumps and the diesel generators, see Figure 18) Then we added the simulation of a water source that would flow into the room. These critical components were marked to send an event to the state simulator when they were flooded with water.

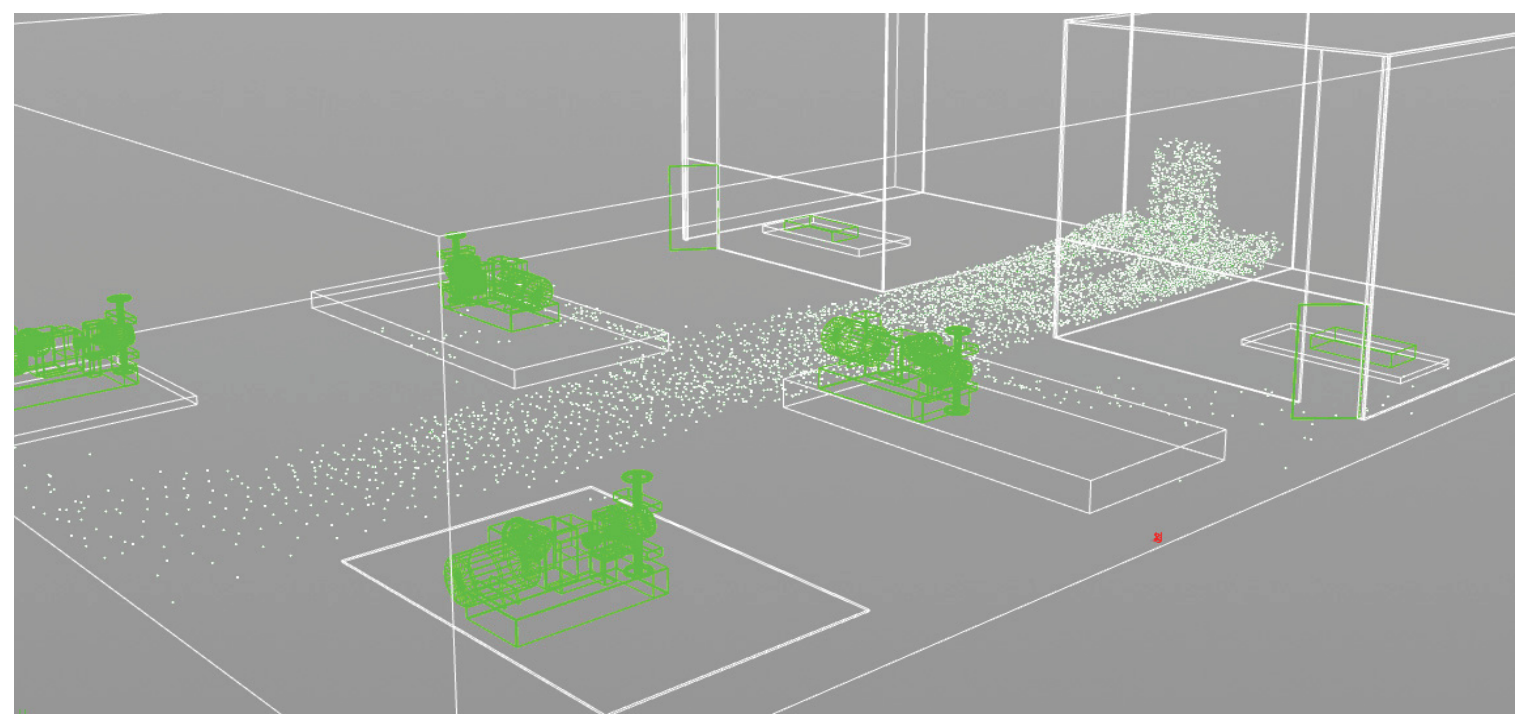

Figure 18 - A 3D model of a simple room with critical components from the Demo model.

On the state simulator side, when these events are received, it triggers the state movement to failure of that component (see Figure 19). Next, we added an initiating event of a tsunami to the "Normal_Op" state which would trigger the movement to a tsunami state which starts 
the 3D simulation; the evaluation of the end states; and possibility of loss of power (see Figure 19). Finally an event transitions to a state which shuts down the 3D simulation and resets the state simulation after a given time frame.

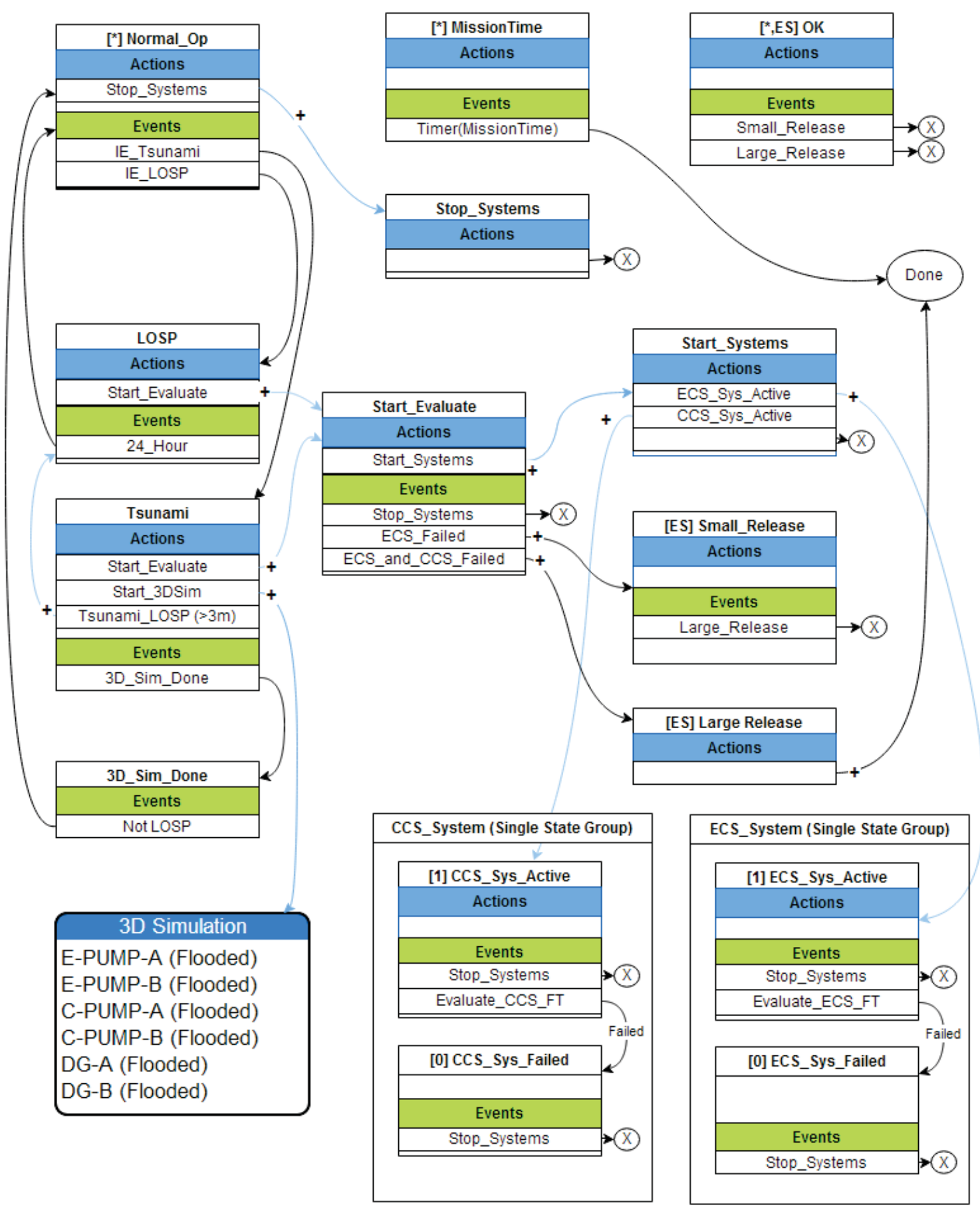

Figure 19 - State diagram model to incorporate 3D simulation results. 
Each simulation that triggers a tsunami flood initiating event also computes flooding flow rates for the component room for the 3D simulation. These flow rates are determined by a sampling of the data given in section 2.1. With the variation of flow rates in each 3D simulation, we get a distribution of the different effects on state simulation.

\subsection{Simulation Results}

\subsubsection{General Tsunami Simulation}

A 3D model of a generic nuclear facility was used in conjunction with the fluid simulation of a large wave to mimic a tsunami. The height of the water at the desired location was measured over time (see Figure 20). This simulation for the tsunami wave was done using the "Neutrino" fluid solver. The Neutrino fluid solver is based on Smooth Particle Hydrodynamics instead of the less accurate grid based estimation approach. The Neutrino fluid solver also factors in advanced boundary handling, and adaptive time stepping to help to increase accuracy and calculation speed. [4] This solver is a custom fluid solver provided to INL by Neutrino Industries.

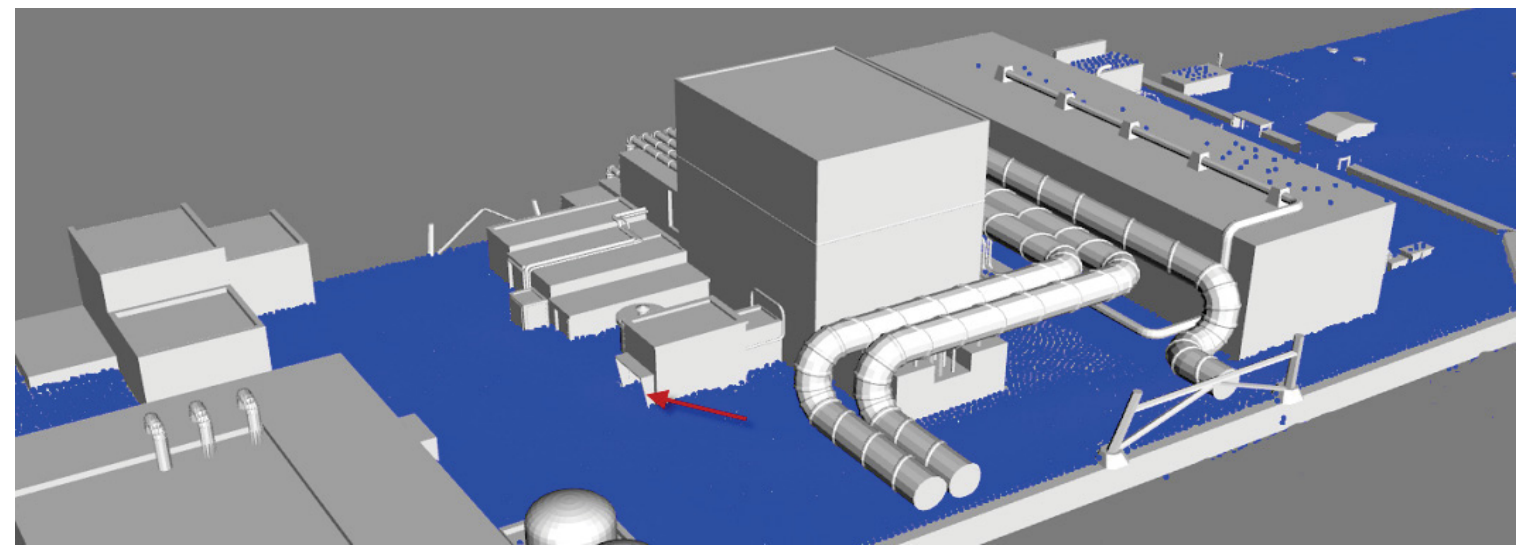

Figure 20 - Measuring the water height at the bay door.

This simulation process was repeated for multiple wave heights to give a range of data points. The water heights at a given point in the simulation can then be converted into a flow rate for further use in more detailed simulations (for example, to represent flooding into multiple support buildings). Using this simulation approach, it was determined that any wave height less than 18 meters (entering the site boundary) resulted in no water reaching the bay door of the facility (located behind the reactor building). 


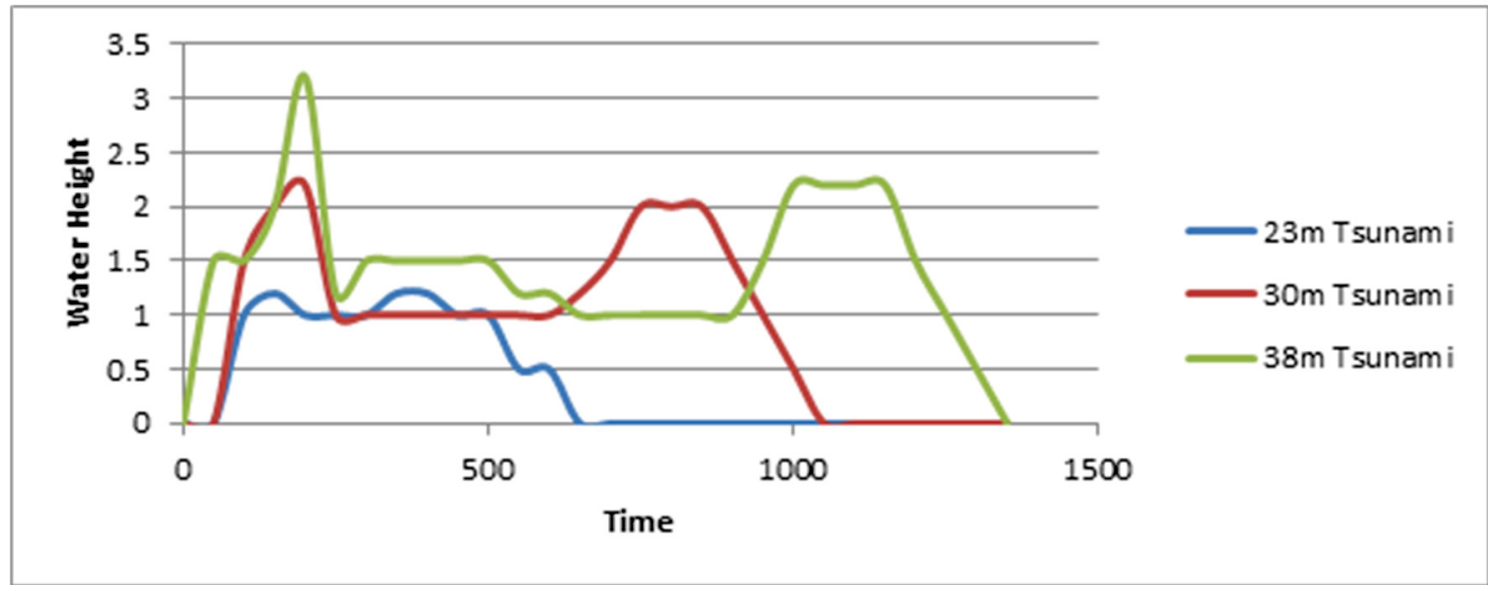

Figure 21 - Measured water heights from different Tsunami waves.

From the data in Figure 21, we can see that the water level rose, dropped a bit, rose again, and then dropped. This behavior of the water height is due to the measuring point being at the back of the facility and, consequently, had flow streams from multiple directions.

\subsubsection{Baseline Results}

Information from the SAPHIRE "Advanced Demo" project was used for the proof of concept simulation model. The only change made was the initiating event frequency for LOSP, where it was reduced from $2.3 / y r$ to $0.1 / y r$ to better reflect industry occurrences ${ }^{1}$. The state-based simulation model was then run 1,0000,000 times to verify an accurate baseline with the SAPHIRE results. The results shown in Table 3 verify state simulation model is statistically equivalent to the SAPHIRE model.

Table 3. Baseline Simulation Results.

\begin{tabular}{|c|c|c|}
\hline End State & SAPHIRE Results & State-based Simulation Results \\
\hline Small Release & $2.53 \mathrm{E}-3 / \mathrm{yr}$ & $2.59 \mathrm{E}-3 / \mathrm{yr}$ \\
\hline Large Release & $7.85 \mathrm{E}-5 / \mathrm{yr}$ & $7.86 \mathrm{E}-5 / \mathrm{yr}$ \\
\hline
\end{tabular}

Additional initiating events for different magnitude tsunami frequencies were then added to this baseline model. One initiating event was added for each of the 1, 10, 100, and 1000 year groupings with frequencies correspond to data given section 2.1. Each time a simulation is run, any tsunami events that could occur are then sampled for wave height data, also given in section 2.1. It is assumed for this analysis that any tsunami height above 3 meters also triggers a LOSP event for the simulation.

The simulations were run with the added tsunami initiating events but without the 3D simulation. The results show the increased failure caused by LOSP triggered by the tsunami and gives another reference point to determine any increase cause just by the 3D simulation.

Table 3. Simulation Results Including Tsunami-cased LOSP Events.

\begin{tabular}{|c|c|c|}
\hline End State & State-based Simulation Baseline & With just Tsunami IE \\
\hline Small Release & $2.59 \mathrm{E}-3 / \mathrm{yr}$ & $3.08 \mathrm{E}-3 / \mathrm{yr}$ \\
\hline Large Release & $7.86 \mathrm{E}-5 / \mathrm{yr}$ & $9.33 \mathrm{E}-5 / \mathrm{yr}$ \\
\hline
\end{tabular}

\footnotetext{
${ }^{1}$ The rate from NUREG-1032 was $0.12 / \mathrm{yr}$ while the rate in NUREG/CR-5496 was $0.058 / \mathrm{yr}$ - for this demonstration we used $0.1 / \mathrm{yr}$.
} 


\subsubsection{D Simulation}

For the 3D simulation, if the wave height is above the $18 \mathrm{~m}$ threshold (for this analysis, we ignored 3D modeling if the tsunami was smaller than $18 \mathrm{~m}$ in order to reduce analysis time), it is translated into water flow rates over a period of time.

Custom flow rates for the sampled wave height are extrapolated in three steps. First, the two nearest simulation data points are determined. Second, for a given point in time a relative point in time is determined from the two known data values. (See Figure 22) Finally, using the flow heights for the relative time points, a percentage between the two values is used to determine the custom flow height (see the equation below).

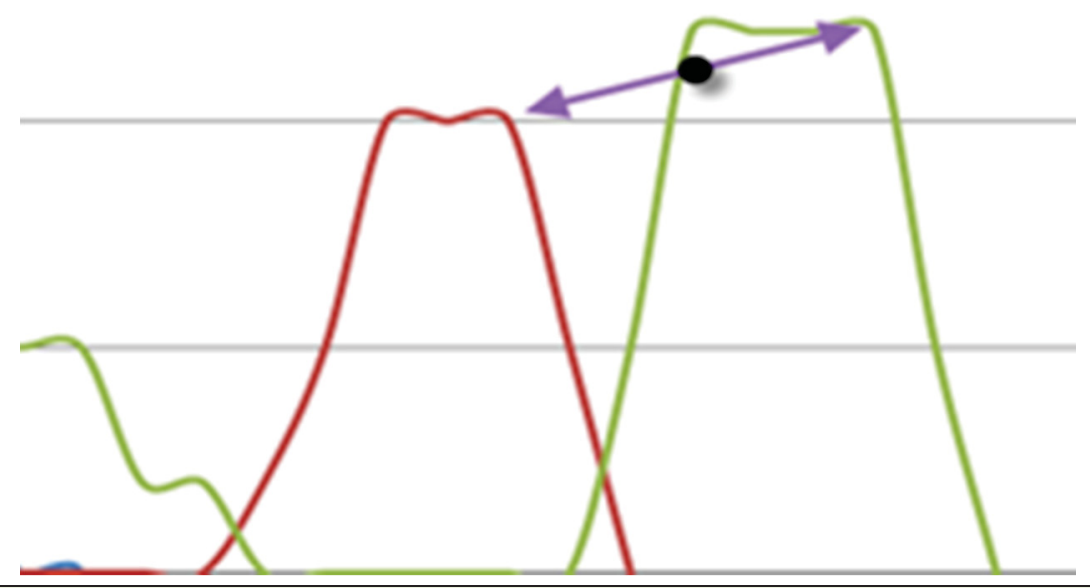

Figure 22 - Relative points and calculated flow for a given time of a sampled wave height.

$$
\operatorname{Flow}(x)=\text { Flow }_{L}+\Delta \text { Flow }\left(\frac{\text { WaveHeight }_{H}-x}{\text { WaveHeight }_{H}-\text { WaveHeight }_{L}}\right)
$$

The next step is to run the state simulation with triggers for the 3D simulation when needed. 


\subsection{Constraints and future activities}

For this initial prototype analyses, a simple room was used for development and initial results. This provided for a good statistical comparison, but was not realistic for an actual facility. A realistic model of a reactor building was created, and can be used, but requires longer simulations times. This more detailed model was used to demonstrate floods entering the building (see Figure 23). When modeling a real facility, the more accurate the 3D model, the more valid the results that would be obtained.

There are several different methods or solvers for water flow simulation, each having its advantages and disadvantages. The one used for the 3D simulation part of the state diagram simulation is a generic "FLIP solver" from the Houdini software. A FLIP solver is a hybrid simulation, combining particle-based and volume-based simulations. However, this solver can over exaggerate water movement and momentum. Using a better fluid solver engine would provide more accurate results when used in a realistic model. With modifications, the Neutrino engine, which was used for the tsunami simulations, could also be used in Houdini for the state simulation process including the water flow inside of the buildings.
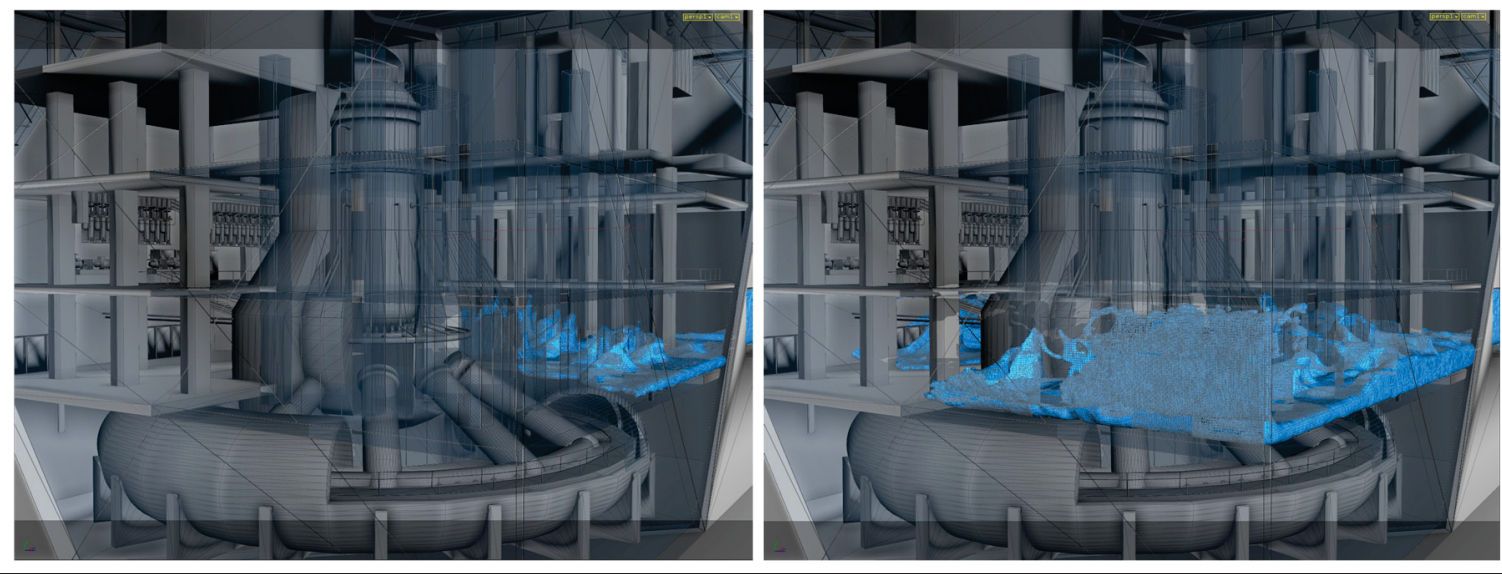

Figure 23 - Example of the flooding simulation for a complicated building structure.

The water heights of the tsunami calculations are an approximation in this analysis. A higher resolution model could be run in order to give a more accurate height. In addition, instead of converting a height to flow rate, modifications to the simulation could be made to calculate the velocity and volume in order to determine a flow rate directly from the tsunami simulation (representing water from the tsunami entering a building). 


\section{Chapter}

3 References

[1] C. Smith, D. Schwieder, S. Prescott, C. Phelan, D. Mandelli, R. Youngblood and K. Vedros, "Small Modular Reactor (SMR) Probabilistic Risk Assessment (PRA) Detailed Technical Framework Specification," Idaho National Laboratory, 2013.

[2] D. Kelly and C. Smith, Bayesian Inference for Probabilistic Risk Assessment: A Practitioner's Guidebook, Springer, 2011.

[3] D. Rasmuson, "A comparison of the small and large event tree approaches used in PRAs," Reliability Engineering and System Safety, vol. 37, pp. 79-90, 1992.

[4] N. Akinci, M. Ihmsen, G. Akinci, B. Solenthaler and M. Teschner, "Versatile Rigid-Fluid Coupling for Incompressible SPH," ACM Transactions on Graphics (Proc. SIGGRAPH 2012), vol. 31, no. 4, 2012. 